\title{
Hysteretic Behaviour of Stockbridge Dampers: Modelling and Parameter Identification
}

\author{
Francesco Foti $(\mathbb{D}$ and Luca Martinelli \\ Department of Civil and Environmental Engineering, Politecnico di Milano, Piazza L. da Vinci 32, 20133 Milano, Italy \\ Correspondence should be addressed to Francesco Foti; francesco.foti@polimi.it
}

Received 20 December 2017; Revised 5 April 2018; Accepted 12 April 2018; Published 20 May 2018

Academic Editor: Yuri Vladimirovich Mikhlin

Copyright (c) 2018 Francesco Foti and Luca Martinelli. This is an open access article distributed under the Creative Commons Attribution License, which permits unrestricted use, distribution, and reproduction in any medium, provided the original work is properly cited.

\begin{abstract}
Stockbridge dampers are widely used to mitigate the vortex-induced vibrations of overhead electrical line conductors and of many other cable or cable-like structures exposed to the action of wind. Aim of this work is to develop a simple, but accurate, mechanical model of a Stockbridge damper to use in the assessment of such structures vibrations. The model is based on a beam-like description of the messenger cable and on a nonlinear formulation of the cross sections cyclic bending behaviour. At the cross-sectional level, the mechanical behaviour of the messenger cable is reproduced with the classic Bouc-Wen hysteretic model, which has been recognized as adequate to represent the local behaviour mainly controlled by interwire sliding processes. The Bouc-Wen model parameters are identified from experimental results available in literature and used at the local (cross-sectional) level to characterize the mechanical behaviour of the messenger cable. The descending global behaviour of the Stockbridge damper compares very favourably with the experimental results in terms of the impedance function at the clamp and allows for the confident use of the proposed model inside the assessing process of full power lines. The important role of the end zones of the messenger cable is highlighted. In these zones, a boundary layer like transition is found to occur for the bending stiffness of the messenger cable cross section. This largely affects the global behaviour of the Stockbridge damper. The length of these zones complements the model parameters.
\end{abstract}

\section{Introduction}

Stockbridge dampers are widely used to mitigate the vortexinduced vibrations of overhead electrical line conductors [15], stay cables [6], and many other cable or cable-like structures exposed to the action of wind, such as the counterweight tension bars of cranes [7].

Invented by Stockbridge in 1925 [8] and subsequently modified by many other researchers (see, e.g., [9]), these dampers are cheap, reliable, and easy to install. The most common type of Stockbridge dampers is made of a rigid clamp, a short metallic strand, also known as "messenger cable," and two inertial bodies attached at the end sections of the strand. The rigid clamp allows connecting the Stockbridge damper along the span of a structural cable, to behave as a secondary dynamic system providing additional dissipation to the structure. Whenever the damper is actuated by the motion of the clamp, the two sides of the messenger cables basically behave as flexible cantilevers with lumped translational and rotational masses at their ends and two principal vibration modes. The normal modes of the messenger cables are, respectively, related to the translation and to the rotation of the inertial bodies attached at their end sections (see, e.g., [10-12]).

Differently than classic, linearly damped, tuned mass vibration absorber [13], the dynamic response of Stockbridge dampers is nonlinear and hysteretic, due to the peculiar bending behaviour of the messenger cable. Relative displacements between the wires of the cable, indeed, can occur during flexural vibrations, depending on the value of the vibration amplitude. These internal sliding phenomena are associated with frictional dissipation, which makes the dynamic response of the damper inherently nonlinear. In particular, both the damping and the dynamic stiffness of the damper turn out to be a function of the vibration amplitude of the support (i.e., the clamp); see, for example, [14]. 
The nonlinear behaviour of the messenger cable is a characteristic feature of the Stockbridge dampers, which makes them a very attractive and effective option to mitigate wind-induced vibrations of cable structures. The nonlinearity, indeed, allows circumventing the detuning effects, typical of linear tuned mass dampers, which can be due to time variations both of the mechanical properties of the damping device and of the operating conditions [15].

Notwithstanding the capital influence in determining the global characteristics of the Stockbridge damper, however, only few models can be found in the literature which specifically address the issue of the characterization of the hysteretic bending behaviour of the messenger cable.

Pivovarov and Vinogradov [16] modelled the messenger cable as an equivalent single-degree-of-freedom system, with restoring forces defined through the phenomenological hysteretic model proposed by Bouc [17]. As a major drawback, the pioneering work by Pivovarov and Vinogradov allows reproducing only a single mode of the messenger cable, due to the single-degree-of-freedom assumption.

More recent formulations are based on the EulerBernoulli beam model to achieve a more realistic description of the modal properties of the system. Sauter and Hagedorn $[18,19]$ modelled the messenger cable as a nonlinear beam with a cross-sectional moment-curvature diagram described through the Masing hysteretic model. The constitutive parameters of the model are identified from the results of static tests. The dynamic response of the system is then studied by developing an ad hoc numerical procedure to circumvent numerical difficulties arising from the nonsmooth character of the Masing model. The predictions of the numerical model are compared with experimental results obtained by testing a Stockbridge damper prototype mounted on a shaker table, showing an encouraging agreement. Nonlinear models based on finite element beam elements have been adopted by Langlois and Legeron [14] and Barbieri et al. $[20,21]$. In both cases, phenomenological hysteretic models are adopted and the constitutive parameters are identified from the results of static or dynamic experimental tests.

A full-scale nonlinear finite element model based on 3D solid elements has been recently developed by Luo et al. [22]. The 3D finite element approach allows introducing a detailed model for the local contact conditions between the wires of the messenger cables as well as between the clamp and the messenger cable, allowing appreciating how these complex local phenomena play a key role in the determination of the overall dynamic characteristics of the Stockbridge damper. In spite of their possible accuracy, however, full-scale nonlinear 3D finite element models are challenging to setup and require a very careful calibration, especially when in presence of many nonlinear contact interfaces.

The aim of this work is to develop a simple, but accurate, mechanical model of a Stockbridge damper to use in the assessment of the vibrations of overhead electrical line conductors or of other cable-like structures, exposed to the action of wind.

The proposed model is based on a beam-like description of the messenger cable and on a nonlinear formulation of the cross sections cyclic bending behaviour.
At the cross-sectional level, the mechanical behaviour of the messenger cable is reproduced with the classic Bouc-Wen hysteretic model, which has been recognized as adequate to represent the local behaviour mainly controlled by interwire sliding processes. The parameters of the Bouc-Wen model are identified from experimental results available in literature and used to characterize the mechanical behaviour of the messenger cable. The descending global behaviour of the damper is compared with experimental results in terms of the impedance function at the clamp.

On the global behaviour, an important role is played by the end zones of the messenger cable, where a boundary layer like transition is found for the bending stiffness of the messenger cable cross section. The length of these zones complements the model parameters.

\section{Equations of Motion of Stockbridge Dampers}

Stockbridge dampers are typically composed of a metallic clamp, a short stretch of a stranded steel cable, which is also known as "messenger cable," and two shaped inertial bodies (see Figure 1(a)). The clamp is commonly made of lightweight aluminium alloys and can be directly cast on the messenger cable or attached to it through compression. In both cases, the manufacturing process ensures the continuity between the clamp and metallic strand. The inertial bodies are usually made of steel or Zinc-Aluminium alloys and can be manufactured in different shapes, the most popular of which are by far the "bell" and the "fork" shape [9]. As it is sketched in Figure 1(a), the damper inertial bodies are connected to the extremities of the messenger cable and can be characterized by different sizes and inertial properties, for a given design shape. As per the clamp, different strategies have been devised by the producers to rigidly connect the damper inertial bodies to the messenger cable (see, e.g., [22]).

Taking into account the manufacturing process, hence, relative displacements at the interface sections $A_{i}(i=1,2)$ in Figure 1(a) can be assumed as strictly equal to zero, and, by neglecting the mass of the messenger cable, the Stockbridge damper can be modelled as a discrete dynamic system made of three rigid bodies connected by means of two flexible massless cantilevers.

As a matter of fact, the Stockbridge damper can be regarded as a "tuned mass vibration absorber" [13], designed to mitigate the effects of vortex-induced vibrations on the overhead electrical conductors and their interconnected equipment. The clamp of the damper rigidly follows the motion of the vibrating conductor and is mainly subjected to a planar motion, resulting from the superposition of the vertical translation $w_{c}(t)$ and rotation $\varphi_{c}(t)$ of the clamp, where the symbol $t$ denotes the time (see also Figure 1(b)). Due to the inherent flexibility of the messenger cable and the kinematic constraint provided by the clamp, the two sides of the damper basically behave as uncoupled planar cantilevers, with inertial bodies at their ends providing lumped translational and rotational masses, and subjected to a motion of the support. Within this context, the motion 


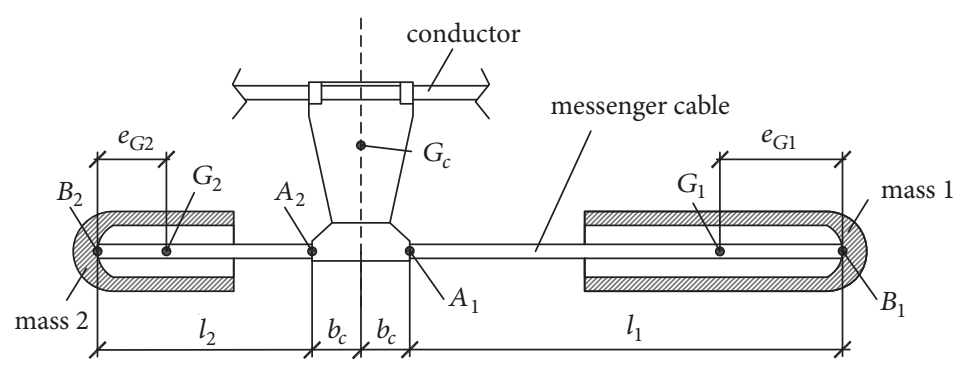

(a)

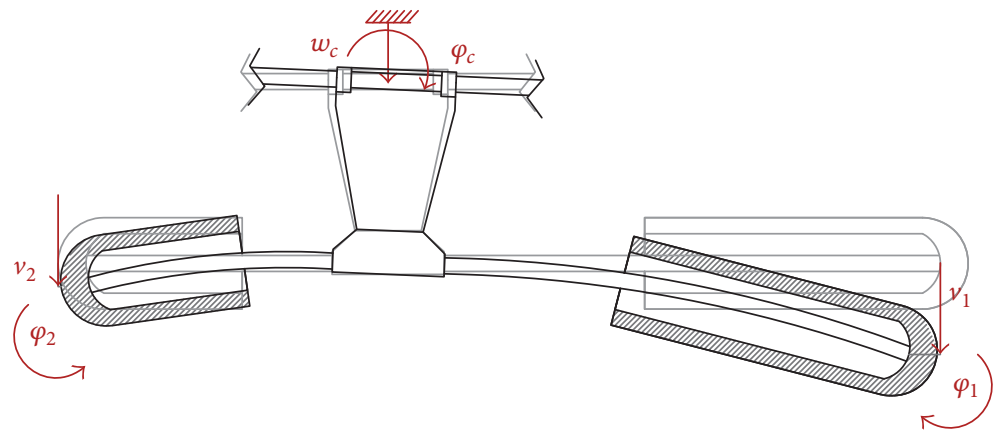

(b)

FIGURE 1: (a) Schematic representation of a Stockbridge damper. (b) Kinematics of the damper.

of the $i$ th $(i=1,2)$ inertial body of the damper, as it is customary in the modelling of Stockbridge dampers (see, e.g., [11]), can be completely described by specifying the vertical displacement $w_{G i}(t)$ of its centroid and the rotation $\varphi_{i}(t)$. It is also convenient to introduce for each side of the messenger cable the relative transverse displacement, $v_{i}(t)$, of the tip with respect to the clamped sections $A_{i}$.

Under the assumption of small displacements and rotations, the vertical translation of the centroid of the $i$ th inertial body (see also Figure 1(b)) can be expressed as

$$
w_{G i}=v_{i}-e_{G i} \varphi_{i}+w_{c}+\varepsilon_{i} b_{c} \varphi_{c}, \quad \text { with: } \varepsilon_{i}=(-1)^{i-1},
$$

where $i=1,2, e_{G i}$ is the distance between the centroid of the $i$ th inertial body and the tip section $B_{i}$ of the messenger cable, and $b_{c}$ is the characteristic length of the clamp highlighted in Figure 1(a).

In the following, the dynamic response of the damper to a generic input motion of the clamp will be investigated.

Accordingly, in Section 2.1 the equations of motion of each inertial body of the damper are first derived for a generic combination of translation $\left(w_{c}\right)$ and rotation $\left(\varphi_{c}\right)$ of the clamp. To this aim, a classic variational formulation for nonholonomic discrete dynamic systems is adopted, in order to properly account for the characteristic inelastic bending behaviour of the messenger cable. Then, starting from the knowledge of the motion of the damper inertial bodies, general closed-form expressions to evaluate the forces acting at the clamp of the damper are obtained in Section 2.2.

2.1. Equations of Motion of the Damper Inertial Bodies. Given the motion of the clamp, the generic dynamic configuration of the $i$ th $(i=1,2)$ inertial body of the Stockbridge damper can be described by introducing two Lagrangian coordinates, such as the relative displacement, $v_{i}$, with respect to the clamped section $\left(A_{i}\right)$ of the end section $\left(B_{i}\right)$ of the messenger cable, $v_{i}$ and the rotation of the inertial body, $\varphi_{i}$. Within this context, the equations of motion of the damper inertial body can be conveniently obtained by means of Hamilton's variational principle for non-holonomic discrete dynamic systems (see, e.g., $[23,24]$ ). By denoting as $t_{1}$ and $t_{2}$, two generic time instants such that $t_{2}>t_{1}$, the following stationarity conditions hold true:

$$
\int_{t_{1}}^{t_{2}}\left(\delta T_{i}+\delta W_{i}\right) d t=0
$$

where $\delta T_{i}$ is the total variation of the kinetic energy of the $i$ th inertial body, while $\delta W_{i}$ is the work done by the restoring forces exerted by the messenger cable on the $i$ th inertial body for a virtual variation of the Lagrangian coordinates $v_{i}$ and $\varphi_{i}$.

By accounting for both the translational (mass: $m_{i}$ ) and the rotational inertia (mass moment of inertia with respect to the centroid: $I_{G i}$ ) of the $i$ th inertial body of the damper, the kinetic energy $T_{i}$ can be expressed as

$$
T_{i}=\frac{1}{2} m_{i} \dot{w}_{G i}^{2}+\frac{1}{2} I_{G i} \dot{\varphi}_{i}^{2},
$$

where the dot denotes the derivative with respect to the time and $w_{G i}$ is the vertical displacement of the centroid (point $G_{i}$ in Figure 1(a)). By recalling the kinematic equation (1), the 


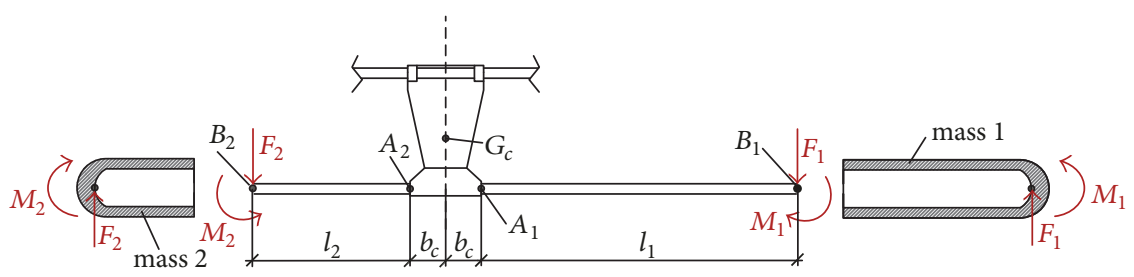

(a)

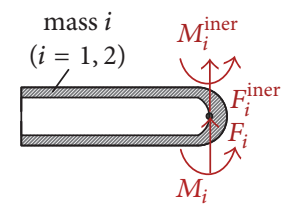

(b)

FIgURE 2: (a) Generalized restoring forces at the end sections of the messenger cables. (b) Dynamic equilibrium of the $i$ th damper mass.

kinetic energy (3) can be also rewritten as a function of the first time derivatives of the Lagrangian coordinates $v_{i}$ and $\varphi_{i}$ :

$$
\begin{aligned}
& T_{i}\left(\dot{v}_{i}, \dot{\varphi}_{i}\right)=\frac{1}{2} m_{i}\left(\left(\dot{v}_{i}-e_{G i} \dot{\varphi}_{i}\right)^{2}\right. \\
& \left.\quad+2\left(\dot{v}_{i}-e_{G i} \dot{\varphi}_{i}\right)\left(\dot{w}_{c}+\varepsilon_{i} b_{c} \dot{\varphi}_{c}\right)+\left(\dot{w}_{c}+\varepsilon_{i} b_{c} \dot{\varphi}_{c}\right)^{2}\right)+\frac{1}{2} \\
& \quad \cdot I_{G i} \dot{\varphi}_{i}^{2} .
\end{aligned}
$$

The restoring forces acting on the $i$ th inertial body and workconjugated to the Lagrangian coordinates $v_{i}$ and $\varphi_{i}$ are the shear force $F_{i}$ and the bending moment $M_{i}$ acting at the end section of the messenger cable, as it is schematically depicted in Figure 2(a). Accordingly, the virtual work $\delta W_{i}$ can be expressed as

$$
\delta W_{i}=-F_{i}\left(v_{i}, \varphi_{i}, t\right) \delta v_{i}-M_{i}\left(v_{i}, \varphi_{i}, t\right) \delta \varphi_{i} .
$$

It is worth noting that, due to the hysteretic bending behaviour of the messenger cable, the shear force and the bending moment which appear in (5) can be described, in general terms, by means of two nonlinear and nonholonomic functions of the Lagrangian coordinates, which depend also on the past history of the problem. A suitable model for these restoring forces will be detailed in Section 3. By taking into account (4) and (5) and applying the fundamental lemma of the calculus of variations (see, e.g., [23]), Euler-Lagrange's equations of motion of the $i$ th inertial body can be easily derived from (2) as

$$
\begin{gathered}
\frac{d}{d t} \frac{\partial T_{i}}{\partial \dot{v}}+F_{i}\left(v_{i}, \varphi_{i}, t\right)=0 \\
\frac{d}{d t} \frac{\partial T_{i}}{\partial \dot{\varphi}_{i}}+M_{i}\left(v_{i}, \varphi_{i}, t\right)=0
\end{gathered}
$$

The equations above state the dynamic equilibrium between the generalized restoring forces exerted by the messenger cable on the damper inertial bodies and the generalized inertia forces: $F_{i}^{\text {iner }}=(d / d t)\left(\partial T_{i} / \partial \dot{v}\right)$ and $M_{i}^{\text {iner }}=$ $(d / d t)\left(\partial T_{i} / \partial \dot{\varphi}_{i}\right)$, as it is schematically depicted in Figure 2(b).

By evaluating the derivatives of the kinetic energy (4), (6) can be further rewritten in the following, more expressive, engineering form:

$$
\begin{aligned}
& m_{i} \ddot{v}_{i}(t)-m_{i} e_{G i} \ddot{\varphi}_{i}(t)+F_{i}\left(v_{i}, \varphi_{i}, t\right) \\
& =-m_{i} \ddot{w}_{c}(t)-m_{i} \varepsilon_{i} b_{c} \ddot{\varphi}_{c}(t) \\
& -m_{i} e_{G i} \ddot{v}_{i}(t)+\left(I_{G i}+e_{G i}^{2} m_{i}\right) \ddot{\varphi}_{i}(t)+M_{i}\left(v_{i}, \varphi_{i}, t\right) \\
& =m_{i} e_{G i} \ddot{w}_{c}(t)+m_{i} e_{G i} \varepsilon_{i} b_{c} \ddot{\varphi}_{c}(t) .
\end{aligned}
$$

The motion of each inertial body of the damper, hence, turns out to be controlled by a system of coupled nonlinear second-order differential equations. It can be observed that the coupling between the vertical displacement $v_{i}$ and the rotation $\varphi_{i}$ is due to both the linear inertial terms in (7) and the nonlinear generalized restoring forces, $F_{i}$ and $M_{i}$.

2.2. Generalized Forces Acting at the Damper Clamp. The external force, $F_{c}$, and moment, $M_{c}$, acting at the clamp and work-conjugated to the imposed clamp displacement $w_{c}$ and rotation $\varphi_{c}$, can be evaluated through simple equilibrium considerations, once the motion of each damper inertial body has been determined by integrating the equations of motion (7). Figure 3 shows a schematic representation of all the forces acting on the clamp at a generic instant of time, that is, the external force and moment, $F_{c}$ and $M_{c}$, the shear forces and bending moments exerted by each side of the messenger cable on the clamp, and the generalized inertia forces due to both the translational and the rotational inertia of the clamp: $F_{c}^{\text {iner }}$ and $M_{c}^{\text {iner }}$. The latter can be expressed as $F_{c}^{\text {iner }}=m_{c} \ddot{w}_{c}$ and $M_{c}^{\text {iner }}=I_{G c} \ddot{\varphi}_{c}$, where $m_{c}$ and $I_{G c}$ are, respectively, the mass of the clamp and the mass moment of inertia with respect to its centroid. 


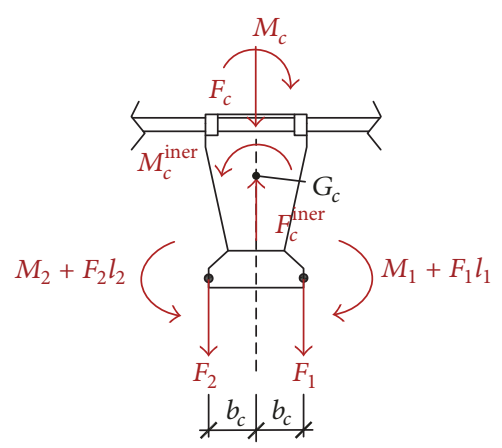

FIGURE 3: Dynamic equilibrium of the clamp.

By imposing the equilibrium of the clamp with respect to both the vertical translation and the rotation, the external force $F_{c}$ and moment $M_{c}$ can be evaluated as

$$
\begin{aligned}
F_{c} & =F_{c}^{\text {iner }}-\left(F_{1}+F_{2}\right) \\
M_{c} & =M_{c}^{\text {iner }}+M_{2}-M_{1}+F_{2}\left(l_{2}+b_{c}\right)-F_{1}\left(l_{1}+b_{c}\right) .
\end{aligned}
$$

By taking into account the equations of motion of the damper inertial bodies (7), (8) can be further rewritten as

$$
\begin{aligned}
F_{c}= & \left(m_{1}+m_{2}+m_{c}\right) \ddot{w}_{c}+\left(m_{1}-m_{2}\right) b_{c} \ddot{\varphi}_{c}+m_{1} \ddot{v}_{1} \\
& +m_{2} \ddot{v}_{2}-m_{1} e_{G 1} \ddot{\varphi}_{1}-m_{2} e_{G 2} \ddot{\varphi}_{2} \\
M_{c}= & \left(m_{1} l_{1}^{*}-m_{2} l_{2}^{*}\right) \ddot{w}_{c}+\left(I_{G c}+m_{1} b_{c} l_{1}^{*}+m_{2} b_{c} l_{2}^{*}\right) \ddot{\varphi}_{c} \\
& +m_{1} l_{1}^{*} \ddot{v}_{1}-m_{2} l_{2}^{*} \ddot{v}_{2}+\left(I_{G 1}-m_{1} e_{G 1} l_{1}^{*}\right) \ddot{\varphi}_{1} \\
& -\left(I_{G 2}-m_{2} e_{G 2} l_{2}^{*}\right) \ddot{\varphi}_{2},
\end{aligned}
$$

where $l_{i}^{*}=l_{i}+b_{c}-e_{G i}, \quad i=1,2$.

Equations (9) allow us to clearly appreciate all the inertial contribution to the generalized forces, acting at the clamp of the damper, coming from the motion of the clamp and of the damper inertial bodies.

For the special case of symmetric Stockbridge dampers, which is often encountered in practical applications, the general equations (9) can be rewritten as

$$
\begin{aligned}
F_{c} & =\left(2 m_{d}+m_{c}\right) \ddot{w}_{c}+2 m_{d} \ddot{v}_{d 2}-2 m_{d} e_{G d} \ddot{\varphi}_{d} \\
M_{c} & =\left(I_{G c}+2 m_{d} b_{c} l_{d}^{*}\right) \ddot{\varphi}_{c},
\end{aligned}
$$

where $m_{d}, I_{G d}$, and $e_{G d}$ are the relevant inertial and geometric properties of the damper inertial bodies, $l_{d}^{*}$ is defined as $l_{d}^{*}=$ $l_{1}+b_{c}-e_{G 1}=l_{2}+b_{c}-e_{G 2}$, and $v_{d}$ and $\varphi_{d}$ are, respectively, the relative vertical displacement and the rotation of the damper inertial bodies $\left(v_{1}=v_{2}=v_{d}\right.$ and $\varphi_{1}=\varphi_{2}=\varphi_{d}$, due to the symmetry of the system).

\section{Hysteretic Model of the Messenger Cable}

The messenger cables are short segments of small-diameter spiral steel strands, with total length typically in the range of $30-50 \mathrm{~cm}$. They are made of wires with circular cross section, twisted around a straight core wire, and grouped in one or two concentric layers. The centerline of the external wires can be modelled as a circular helix, wrapped around the centerline of the strand, with radius $R$ and pitch $P$. The constant angle between the tangent vector to the wire centerline and the axis of the strand can be evaluated as $\alpha=\arctan (2 \pi R / P)$ and is commonly referred to as the "lay angle" of the wire. Typical values of the lay angle for messenger cables are in the range of $10^{\circ}-30^{\circ}$. Different layers are typically made of wires wrapped with opposite directions to enhance the stability of the cable (see also [25], for further details on the geometric modelling of spiral strands).

The wires of the messenger cable are clenched together, such that even if the strand is axially unloaded each wire is in radial contact with the ones belonging to an adjacent layer or to the core (for the wires in the innermost layer). This clenching effect can be recognized as a consequence of two main causes: (1) the kinematic restraints provided by the clamp and the damping inertial bodies; (2) the residual radial contact stresses, which are induced by the manufacturing process and depend both on the complex sequence of operations involved in the winding of the helical wires and on the viscoelastic phenomena characterizing the subsequent storing of the strand (see, e.g., [25-27]). The clenching forces between the wires play a major role on the bending response of metallic strands, as it has been recently shown, for example, in [25].

Whenever a strand is bent, an axial force gradient is generated along the wires, giving them the trend to slip with respect to the neighbouring ones. This relative displacement is counteracted by friction forces, acting on the internal contact surfaces between the wires belonging to adjacent layers (or between the wires of the innermost layer and the core). According to the classic Amontons-Coulomb friction model, the interwire friction forces increase linearly with the radial contact forces, which in slack cables only depend on the previously discussed clenching effects due to the residual stresses and to the boundary conditions. The axial force gradient along the wires can be increased, up to the point to overcome the value of the interwire friction forces, by increasing the value of the bending curvature of the strand. This leads to an unbalanced condition determining the sliding of the wire and the freezing of the axial force gradient (see, e.g., $[25,28]$ ).

Figure 4 depicts a typical moment-curvature $(M-\chi)$ for the cross section of a metallic strand (see, e.g., [29]). A physically sound interpretation of the nonlinear and nonholonomic bending behaviour depicted in Figure 4 can be obtained based on the simple but well-established [25, 28-30] friction model previously described.

Initially, the friction forces are large enough to prevent any relative sliding between the wires. Since all the wires of the strand are stuck together, this limit kinematic condition is usually referred to as the "full-stick" state of the strand. The cross section behaves as a planar rigid body according to the classic Euler-Bernoulli beam model and the tangent bending stiffness (defined as $E I_{\tan }=\partial M / \partial \chi$ ) is at a maximum value, that is, $E I_{\tan }=E I_{\max }$. 


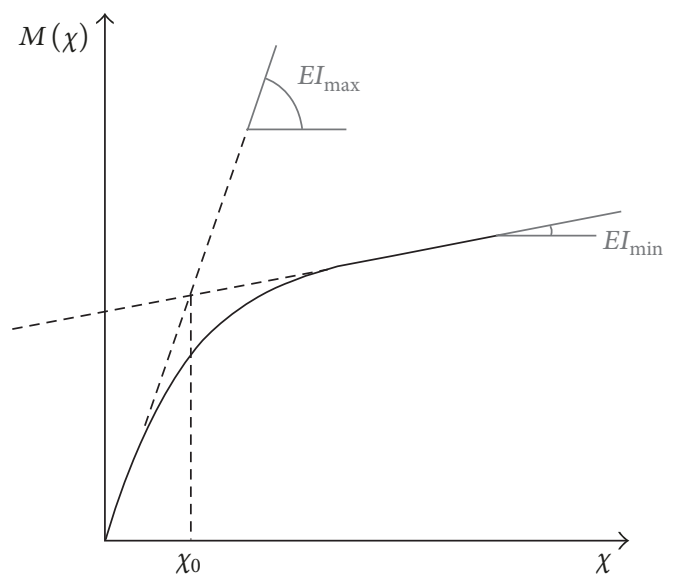

FIgURE 4: Typical bending moment curvature diagram of metallic strands.

By increasing the value of the bending curvature, the wires progressively start to slip and the tangent stiffness $E I_{\text {tan }}$ of the cable gradually decreases up to reach the minimum value: $E I_{\tan }=E I_{\min }$, when all the wires are in the slipping state. In this limit condition, usually referred to as the "full-slip" state, the strand basically reacts as a bundle of individually bent wires to any increment of curvature.

Several expressions have been proposed in the literature to evaluate the maximum and minimum value of the tangent stiffness of metallic strands. Since they start from very similar mechanical assumptions, most of them lead to very close results (see, e.g., $[28,31])$. The following expressions, previously developed by the authors in [28] to model the planar bending of a multilayer metallic strand, will be adopted in this work to obtain a theoretical estimate of the stiffness values $E I_{\min }$ and $E I_{\max }$ of the messenger cable:

$$
\begin{aligned}
& E I_{\min }=\sum_{j=0}^{m} n_{j} \cos ^{3}\left(\alpha_{j}\right) E I_{w j} \\
& E I_{\max }=E I_{\min }+\sum_{j=0}^{m} \frac{n_{j}}{2} \cos ^{3}\left(\alpha_{j}\right) R_{j}^{2} E A_{w j},
\end{aligned}
$$

where $m$ is the number of the layers; $n_{j}$ is the number of the wires belonging to $j$ th layer; $E A_{w j}$ and $E I_{w j}$ are, respectively, the linearly elastic axial and bending stiffness of the cross section of a wire belonging to $j$ th layer, and $R_{j}$ and $\alpha_{j}$ are the radius and lay angle of layer $j$, respectively. The subscript " 0 " is adopted for the variables which are referred to the wire core of the strand.

It is worth noting that the limit values of the tangent bending stiffness, $E I_{\max }$ and $E I_{\min }$, of the cross section only depend on the mechanical and geometric properties of the wires. On the other hand, the nonlinear transition between the full-stick and full-slip state in the bending momentcurvature diagram of Figure 4 is controlled by the evolution of the relative sliding between wires. A description of this nonlinear transition branch can be obtained from physically based discrete models of the strand; see, for example, [25, $28,30]$. Within these formulations, each wire of the strand is individually modelled as a curved thin rod and the relative sliding between the wires is studied starting from the solution of the frictional stick-slip problem at the internal contact surfaces.

The aforementioned discrete modelling approach can only be fully exploited if a sufficiently accurate description of the radial contact forces between the wires of the strand is available. This normally cannot be achieved in the case of messenger cables. In fact, reliable analytical estimates of the radial contact forces between the wires of the messenger cable are precluded by the lacking of knowledge on the manufacturing process and past history of the strand, together with all the uncertainties inherently related to the mechanical modelling of the local clenching effects in the neighbourhood of the clamp and of the damper inertial bodies.

To circumvent these difficulties, a different, phenomenological, approach is adopted in this work to model the hysteretic bending behaviour of the messenger cable. Accordingly, the moment-curvature law is described by means of the well-known Bouc-Wen hysteretic model (see, e.g., [32, 33]). The five-parameter formulation proposed by Ikhouane et al. [34-36] is herein rewritten in a slightly modified form, which allows for a more intuitive interpretation of the physical meaning of the constitutive parameters of the hysteretic model. By denoting $\chi_{0}$ as the yielding curvature of the approximate bilinear elastic-plastic model depicted in Figure 4, the following equation can be introduced:

$$
M(\chi(t), t)=E I_{\min } \chi(t)+\left(E I_{\max }-E I_{\min }\right) \chi_{0} \eta(t),
$$

where $\eta(t)$ is a hysteretic nondimensional variable. The evolution in time of the hysteretic variable is then described through the following first-order differential equation:

$$
\begin{aligned}
& \dot{\eta}(t)=\frac{1}{\chi_{0}}\left(\dot{\chi}(t)-\sigma|\dot{\chi}(t)||\eta(t)|^{n-1} \eta(t)\right. \\
& \left.\quad+(\sigma-1) \dot{\chi}(t)|\eta(t)|^{n}\right),
\end{aligned}
$$

where $\sigma$ and $n$ are two parameters affecting the shape of the nonlinear transition curve between the two linear regions of the moment-curvature diagram in Figure 4. Some restrictions on the values of the constitutive parameters of the model are imposed by the physical properties of the problem. Indeed, the stiffness terms $\left(E I_{\min }\right.$ and $\left.E I_{\max }\right)$ and the yielding curvature $\chi_{0}$ are such that $E I_{\max }>E I_{\min }>0$, and $\chi_{0}>0$ (see also Figure 4). Furthermore, to ensure the stability and the thermodynamic admissibility of the constitutive equations, the parameters $\sigma$ and $n$ should satisfy the following inequalities: $\sigma \geq 1 / 2$ and $n \geq 1$ (see, e.g., [36]).

By assuming that each section of the messenger cable is characterized by the hysteretic model defined in (12) and (13), the relationship among the generalized displacements $v_{i}$ and $\varphi_{i}$ (with $i=1,2$ ), at the end sections of the messenger cable, and the work-conjugated generalized restoring forces $F_{i}$ and $M_{i}$, (with $i=1,2$ ) (i.e., the generalized forces exerted by the messenger cable on the $i$ th inertial body of the damper) can be consistently defined with a straightforward application of the Principle of Virtual Works (PVW). 


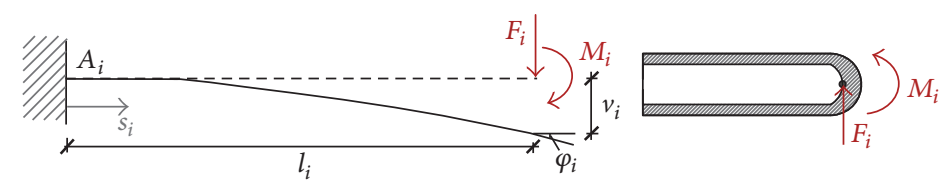

FIGURE 5: Schematic representation of the action of the restoring forces exerted by the messenger cable on the $i$ th inertial body. $F_{i}$ and $M_{i}$ act on the $i$ th side of the messenger cable.

To this aim, it is worth noting that the bending moment distribution along the $i$ th $(i=1,2)$ branch of the messenger cable can be evaluated from the statically determined scheme depicted in Figure 5 (see also Figure 2). Referring to Figure 5 for notation, the following equation can be easily obtained:

$$
M\left(s_{i}, t\right)=M_{i}(t)+F_{i}(t) l_{i}\left(1-\frac{s_{i}}{l_{i}}\right), \quad 0 \leq s_{i} \leq l_{i} .
$$

By neglecting the effects of the shear deformability and considering a generic infinitesimal variation of the external generalized forces acting at the tip of the cable (denoted in the following as $\delta F_{i}$ and $\delta M_{i}$ ) along with a statically admissible variation of the bending moment distribution (denoted in the following as $\delta M\left(s_{i}\right)$ ), the application of the PVW leads to the following equation:

$$
v_{i} \delta F_{i}+\varphi_{i} \delta M_{i}=\int_{0}^{l_{i}} \chi\left(s_{i}\right) \delta M\left(s_{i}\right) d s_{i} .
$$

By taking into account (14) and recalling the arbitrariness of the variations $\delta F_{i}$ and $\delta M_{i}$, (15) can be used to express the tip displacement, $v_{i}$, and rotation, $\varphi_{i}$, as a function of the curvature distribution, $\chi\left(s_{i}\right)$, along the cable. Then, by making explicit the dependency of the curvatures on the generalized forces acting at the tip of the element, the following equations can be derived from (15):

$$
\begin{gathered}
v_{i}\left(F_{i}(t), M_{i}(t)\right) \\
\quad=l_{i} \int_{0}^{l_{i}}\left(\left(1-\frac{s_{i}}{l_{i}}\right) \chi\left(s_{i}, F_{i}(t), M_{i}(t)\right)\right) d s \\
\varphi_{i}\left(F_{i}(t), M_{i}(t)\right)=\int_{0}^{l_{i}}\left(\chi\left(s_{i}, F_{i}(t), M_{i}(t)\right)\right) d s .
\end{gathered}
$$

The above expressions can be used directly within a numerical incremental solution strategy, to evaluate the generalized displacements $v_{i}$ and $\varphi_{i}$, if the time history of the external force, $F_{i}(t)$, and moment, $M_{i}(t)$, are provided in input. In this work, the integrals (16) are evaluated numerically, by means of a Gauss-Lobatto integration scheme. At each integration point, the curvature is first determined for a given increment of the cross-sectional bending moment. To this aim, a Newton-Raphson solver is adopted along with a classic one-step backward Euler algorithm (see, e.g., [37]) to integrate the rate-form constitutive equations (12) and (13). This step of the numerical solution is referred to as crosssectional "state determination."

Equations (16) can be easily solved also for the generalized forces $F_{i}(t)$ and $M_{i}(t)$, if the time history of the displacement, $v_{i}(t)$, and rotation, $\varphi_{i}(t)$, are provided in input. This is a typical problem in the implementation of flexibility-based beam theories formulations (e.g., [38-40]) and a necessary ingredient to numerically solve the nonlinear equations of motion of the $i$ th damper inertial body (7) with classic timestepping schemes, such as the Newmark ones (see, e.g., [37]). In this work, for a given increment of the displacement, $v_{i}(t)$, and rotation, $\varphi_{i}(t),(16)$ are iteratively solved in order to evaluate the corresponding generalized forces with a full Newton-Raphson algorithm. The cross-sectional state determination is performed at each iterative correction step and for all the integration points along the strand, leading to two nested Newton-Raphson algorithms (i.e., both at the (global) element level and at the (local) cross-sectional one). This is a classic solution strategy for flexibility-based beam elements (N-N algorithm in [39]).

\section{Identification of the Model's Parameters}

Being the modelling approach a mechanical one, based on the physics of the Stockbridge damper, the constitutive parameters of the hysteretic model defined in Section 3 can be conveniently identified from the results of quasistatic bending tests on the messenger cable. This is a direct consequence of the rate-independent dissipative behaviour of the cable (see, e.g., [19]), which also is correctly reproduced by the hysteretic Bouc-Wen model (see, e.g., [32]) adopted in this work to characterize the moment-curvature diagram of the cross sections of the cable. If this approach will prove successful, it would open the way to an alternative and easier characterization of the mechanical response of the messenger cable; that can be based on the results for cyclic loading of proved mechanical models of the cable, should these be available.

Although the common industrial practice is mostly based on the dynamic testing of the Stockbridge dampers [41], some results of static bending tests have been also reported in the literature. Usually these tests are based on a very simple experimental setup, made of a short stretch of messenger cable clamped at one end and free at the other one. The free end of the specimen is then subjected to a prescribed transverse displacement at the tip, while recording the corresponding transverse force applied to the strand (or vice versa). The load-displacement curves can be obtained both for monotonically increasing and for cyclically varying transverse displacements (or forces) and assumed as a reference to characterize the overall bending behaviour of the strand (see, e.g., $[10,11])$.

A similar test setup was also adopted by Sauter and Hagedorn [18, 19], which additionally measured the local 
TABLE 1: Geometric characteristics of the messenger cable. The data are from [18].

\begin{tabular}{lcccc}
\hline Layer & Number of wires & Wire diameter, $d(\mathrm{~mm})$ & Helix radius, $R(\mathrm{~mm})$ & Lay angle, $\alpha\left(^{\circ}\right)$ \\
\hline 0 (core wire $)$ & 1 & 2.0 & - & - \\
1 & 6 & 2.0 & 2.0 & 24.69 \\
2 & 12 & 2.0 & 4.0 & 21.44 \\
\hline
\end{tabular}

TABLE 2: Parameters of the proposed cross-sectional hysteretic model identified from the cyclic static test (see also Figure 6).

\begin{tabular}{lcccccc}
\hline Section & Position $s(\mathrm{~m})$ & $E I_{\max }\left(\mathrm{Nm}^{2}\right)$ & $E I_{\min }\left(\mathrm{Nm}^{2}\right)$ & $\chi_{0}\left(\mathrm{~m}^{-1}\right) \times 10^{-2}$ & 4.0 & \\
\hline 1 & 0.005 & 40 & 5.0 & 3.5 & 3.0 & 1 \\
2 & 0.015 & 35 & 32.5 & 2.8 & 2.8 & 1 \\
3 & 0.025 & 30.5 & 2.6 & 2.8 & 1 \\
4 & 0.035 & 25 & 2.4 & 2.3 & 1 \\
5 & 0.05 & 25 & 2.5 & 2.8 & 1 \\
6 & 0.12 & 25 & 2.5 & 2.6 & 1 \\
7 & 0.15 & 25 & 2.5 & 1.8 & 1 \\
8 & 0.18 & 40 & 2.5 & 0.8 & 1 \\
9 & 0.205 & 40 & 2.0 & 0.3 & 1 \\
10 & 0.23 & 0.25 & & 1 \\
11 & & & 1 & 1 \\
\hline
\end{tabular}

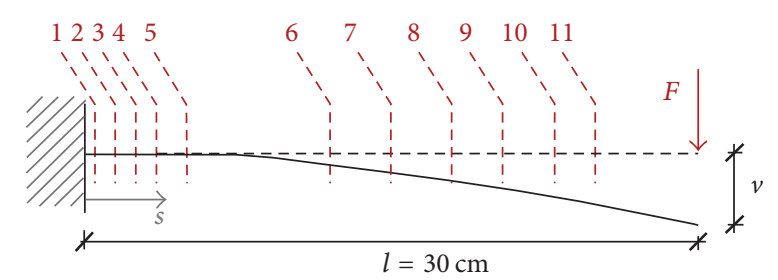

FIGURE 6: Schematic representation of the static experimental test on the messenger cable reported by Sauter and Hagedorn $[18,19]$.

curvatures on several cross sections along the specimen by means of a special arrangement of strain gauges. This refined measurement system allowed the authors to record both the global load-displacement curve and the local momentcurvature diagrams along the messenger cable. Combining both local (i.e., cross-sectional) and global information, the experimental results presented by Sauter and Hagedorn [18, 19] can be conveniently used to identify the parameters and critically assess the validity and limitations of the hysteretic model proposed in the present work for the messenger cables.

The messenger cable tested by Sauter (see, e.g., [18]) was made of two external layers of wires wrapped around a straight core wire. All wires were made of steel and with the same diameter $d=2 \mathrm{~mm}$. All relevant geometric characteristics of the wires are listed in Table 1. The tested specimen, with a length $l=30 \mathrm{~cm}$, was rigidly clamped at one end and subjected to a cyclic transverse displacement, $v$, of the tip. Figure 6 shows a schematic representation of the test setup along with the position of eleven cross sections for which the experimentally determined moment-curvature diagrams are available in [18].
For each cross section, the parameters of the proposed hysteretic cross-sectional moment-curvature relation ((12) and (13)) have been identified and are reported in Table 2. Figures 7-11 show the comparisons between the momentcurvature diagrams measured by Sauter [18] and the ones predicted by the proposed model. After calibration of the parameters, the computed results are in excellent agreement with the experimental ones, clearly highlighting the validity of the proposed phenomenological hysteretic model in reproducing the main characteristic of the physical problem.

Some interesting conclusions on the bending behaviour of the strand can be drawn by analysing the variation along the length specimen of the identified values of the model parameters. First of all, it is worth noting that the nondimensional parameters $\sigma$ and $n$ can be both assumed equal to the unity along the whole length of the cable. In this respect, sections 10 and 11 can be regarded as an exception, with an identified value of $\sigma=10$. However, this markedly different value of the parameter $\sigma$ can also be influenced by the poor quality of the measured moment-diagram curves of sections 10 and 11 (see Figure 11). The impaired quality of the data, on the other hand, can be due to the fact that being the two sections close to the tip of the cantilever they are subjected to small values of bending moment to which it corresponds to a more complex sliding process [42]. Note also that all the values of $\sigma$ in Table 2 correspond to a thermodynamically consistent (Class I, according to [36]) behaviour.

Differently than $\sigma$ and $n$, the identified values of the parameters $E I_{\max }, E I_{\min }$, and $\chi_{0}$ show some clear trends along the length of the cable, as it can be appreciated from Figures 12 and 13 , where they are plotted against the nondimensional abscissa $s / l$, which is, respectively, equal to zero at the clamp 


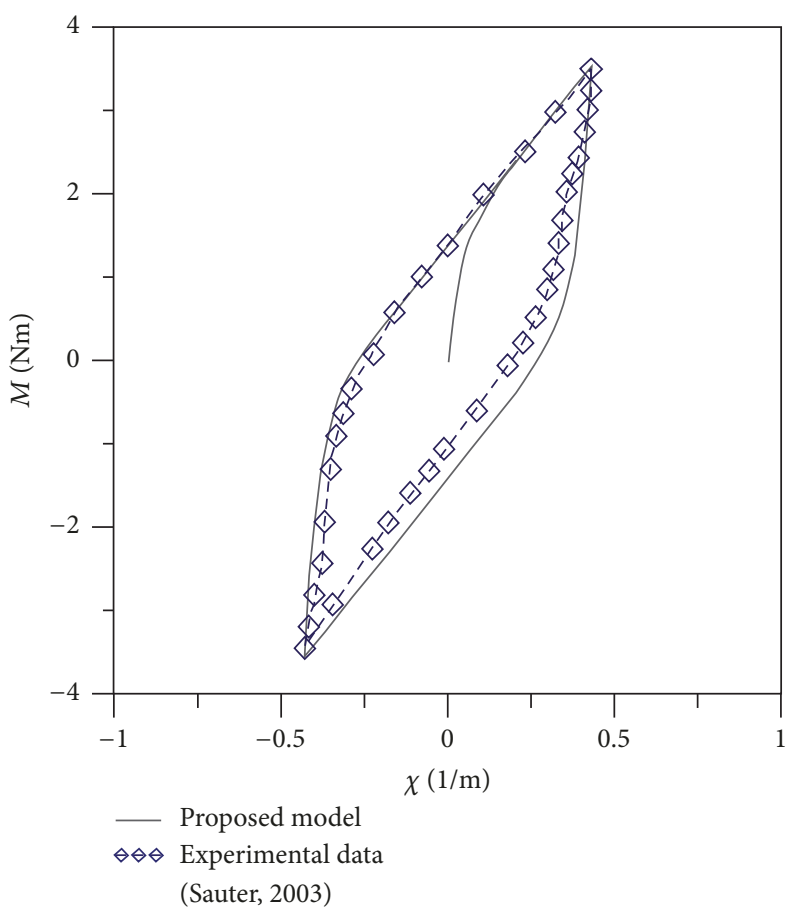

(a)

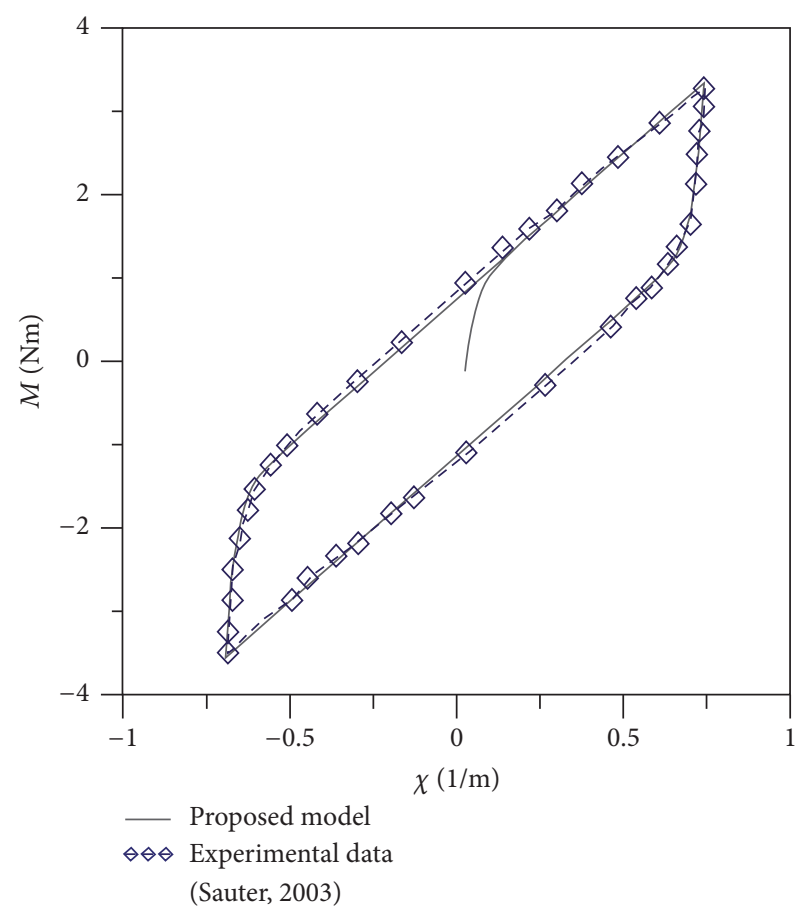

(b)

FIGURE 7: Cross-sectional moment-curvature diagram. Comparison between the proposed model and the experimental data from Sauter [18]. (a) Section 1. (b) Section 2.

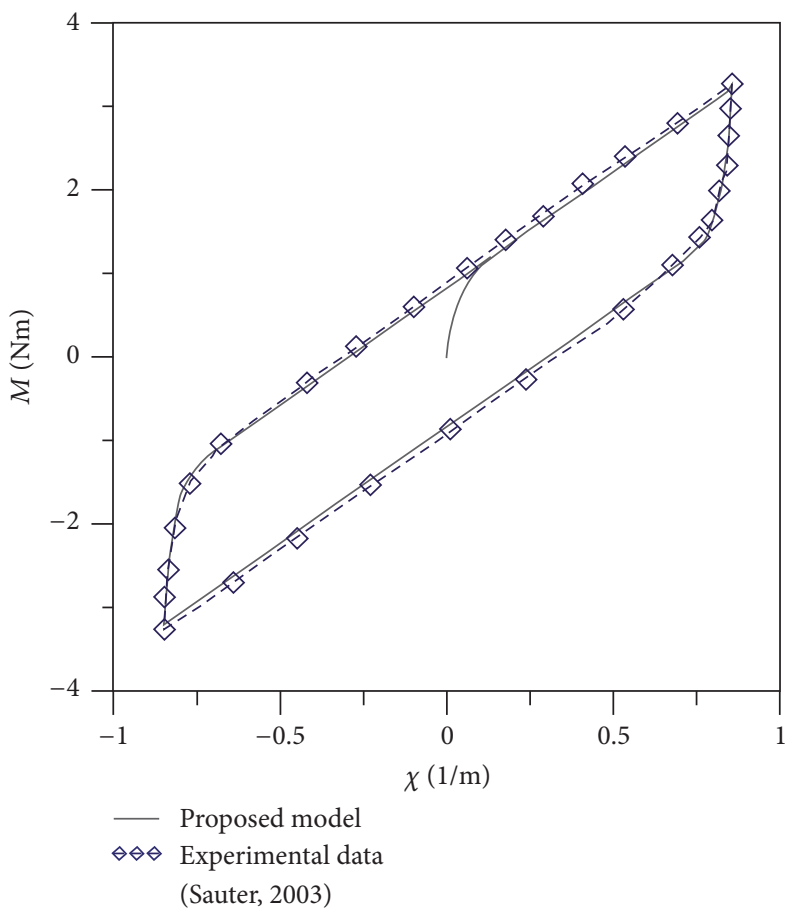

(a)

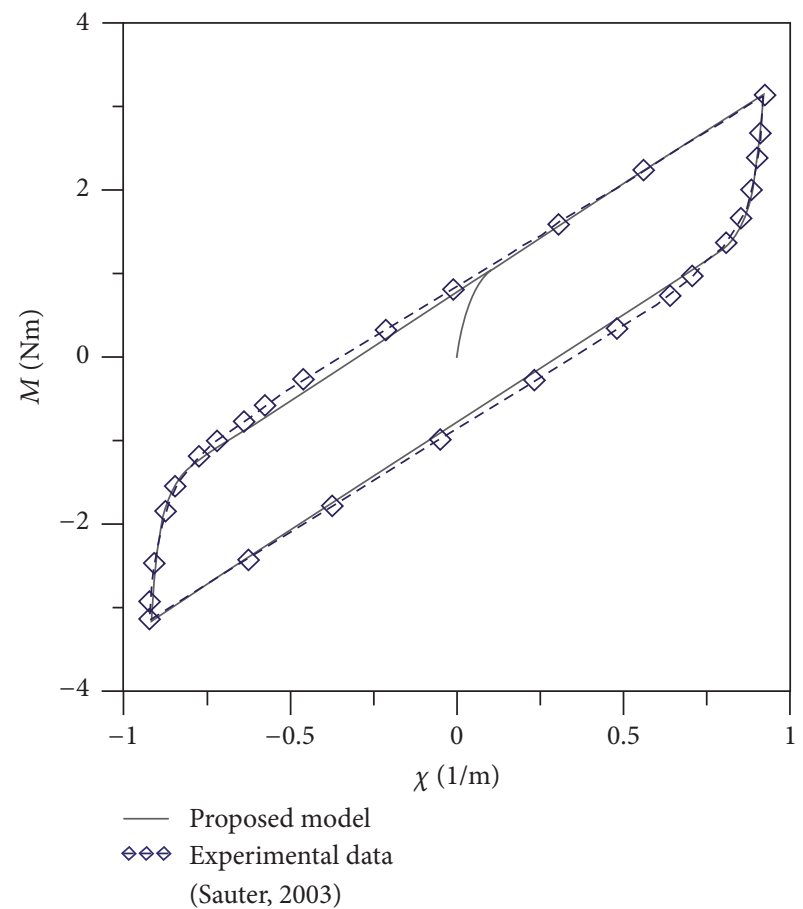

(b)

Figure 8: Cross-sectional moment-curvature diagram. Comparison between the proposed model and the experimental data from Sauter [18]. (a) Section 3. (b) Section 4. 


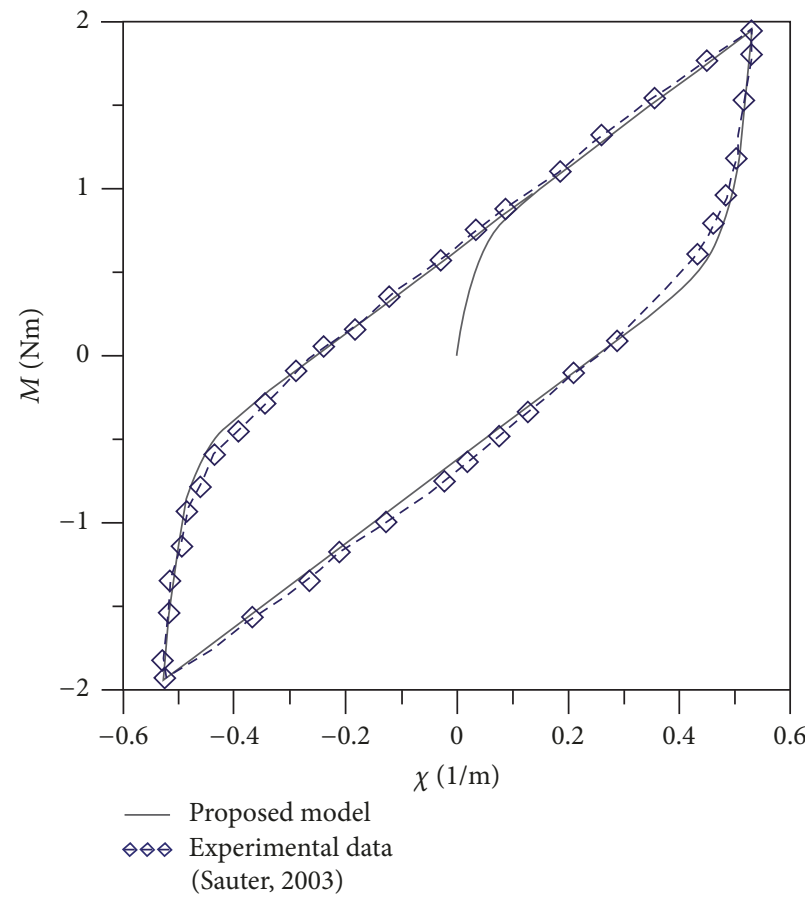

(a)

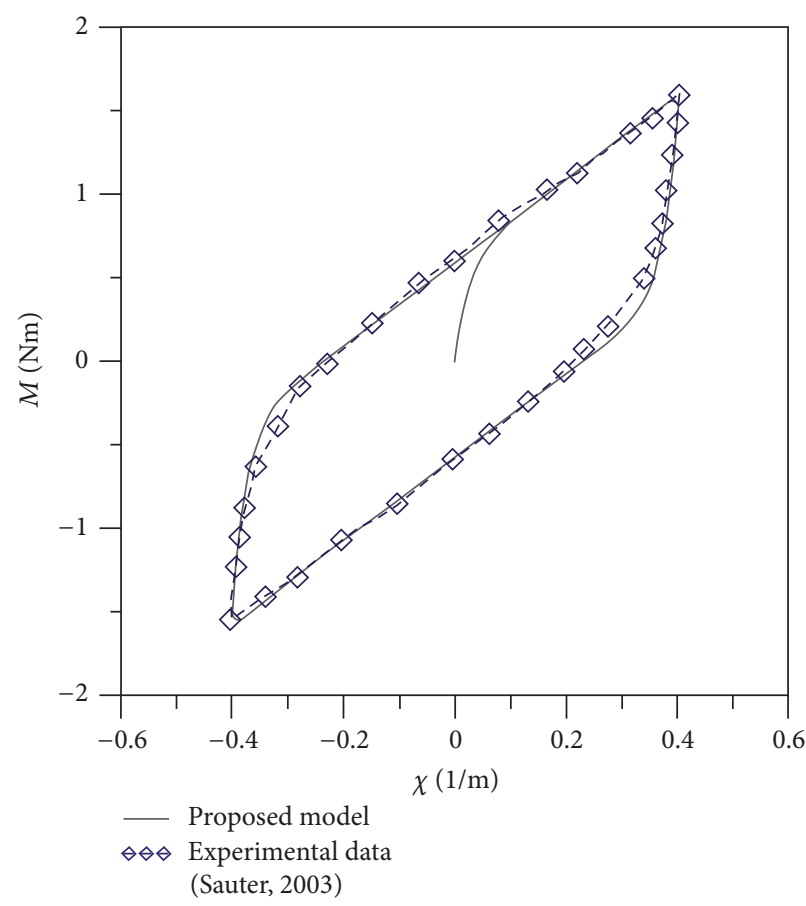

(b)

Figure 9: Cross-sectional moment-curvature diagram. Comparison between the proposed model and the experimental data from Sauter [18]. (a) Section 6. (b) Section 7.

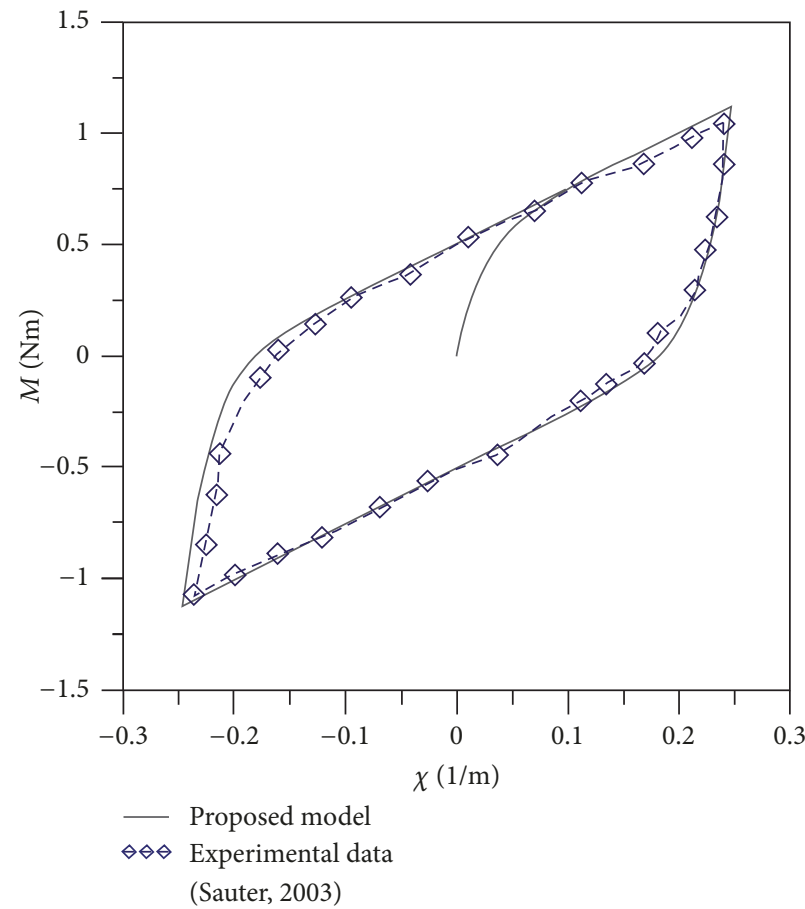

(a)

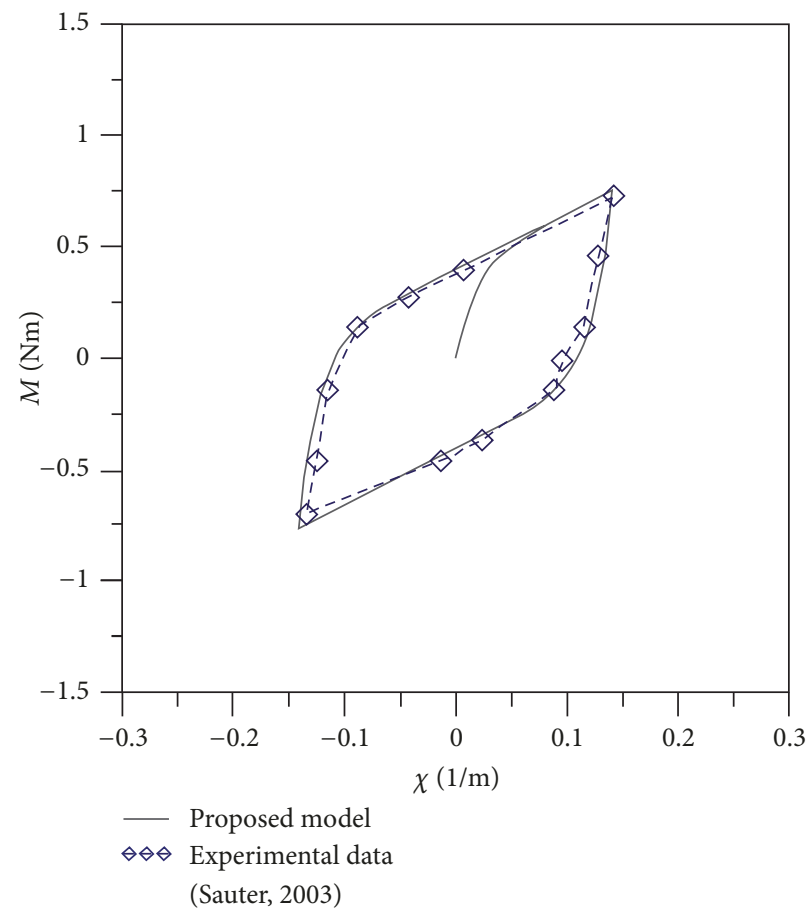

(b)

FIGURE 10: Cross-sectional moment-curvature diagram. Comparison between the proposed model and the experimental data from Sauter [18]. (a) Section 8. (b) Section 9. 


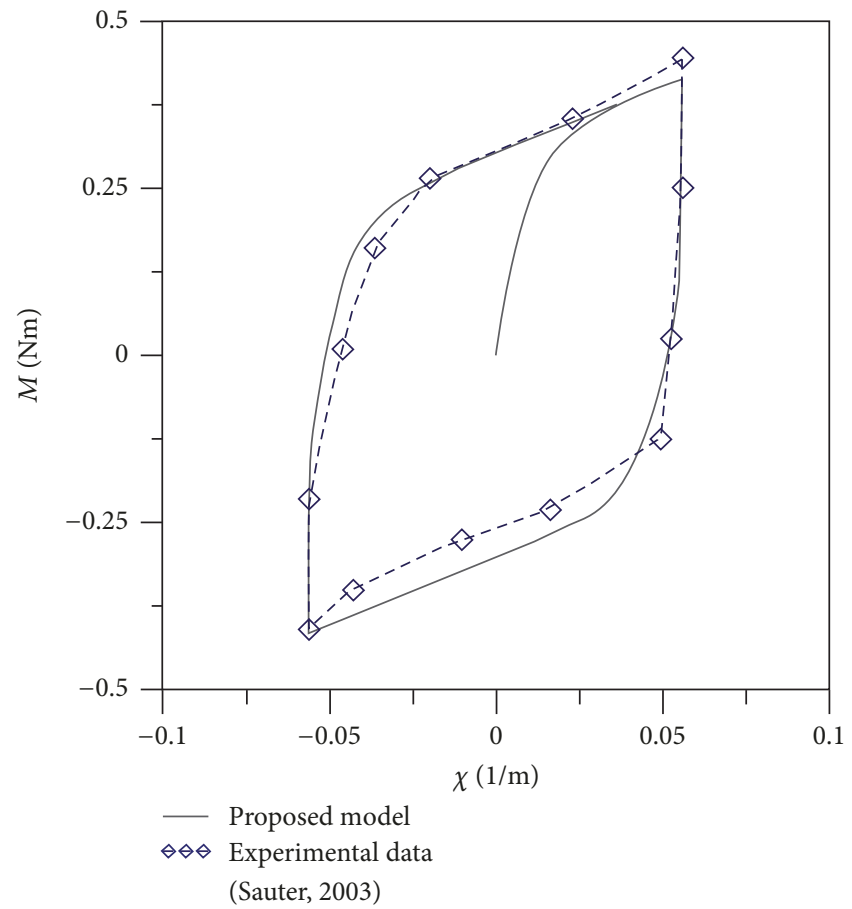

(a)

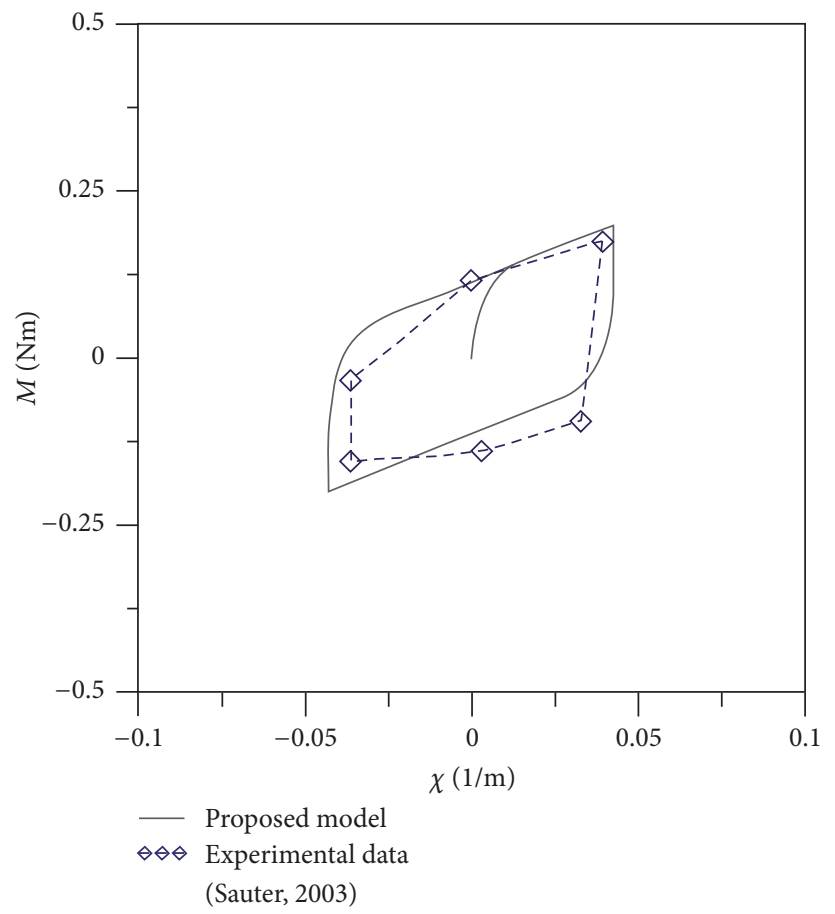

(b)

Figure 11: Cross-sectional moment-curvature diagram. Comparison between the proposed model and the experimental data from Sauter [18]. (a) Section 10. (b) Section 11.

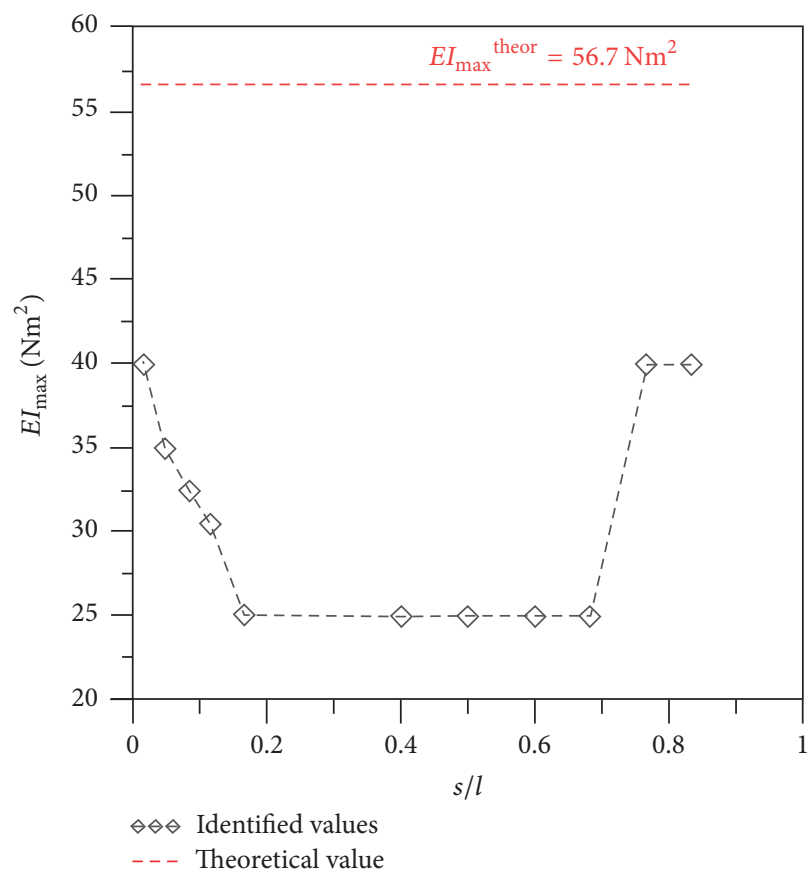

(a)

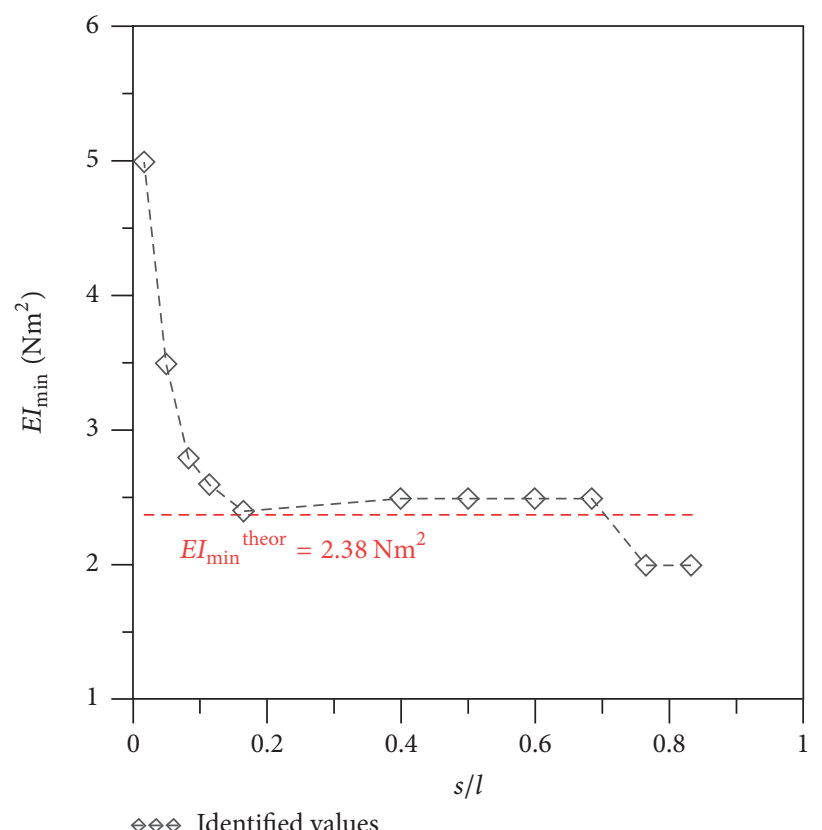

$\diamond \diamond \diamond$ Identified values _. - Theoretical value

(b)

FIGURE 12: Maximum and minimum values of the cross-sectional bending stiffness of the messenger cable. Comparison between the values identified from the static cyclic test and the theoretical values calculated from (11). The values are plotted against the nondimensional arclength coordinate $s / l$ (see also Figure 6). (a) Maximum stiffness value $E I_{\max }$. (b) Minimum stiffness value $E I_{\min }$. 


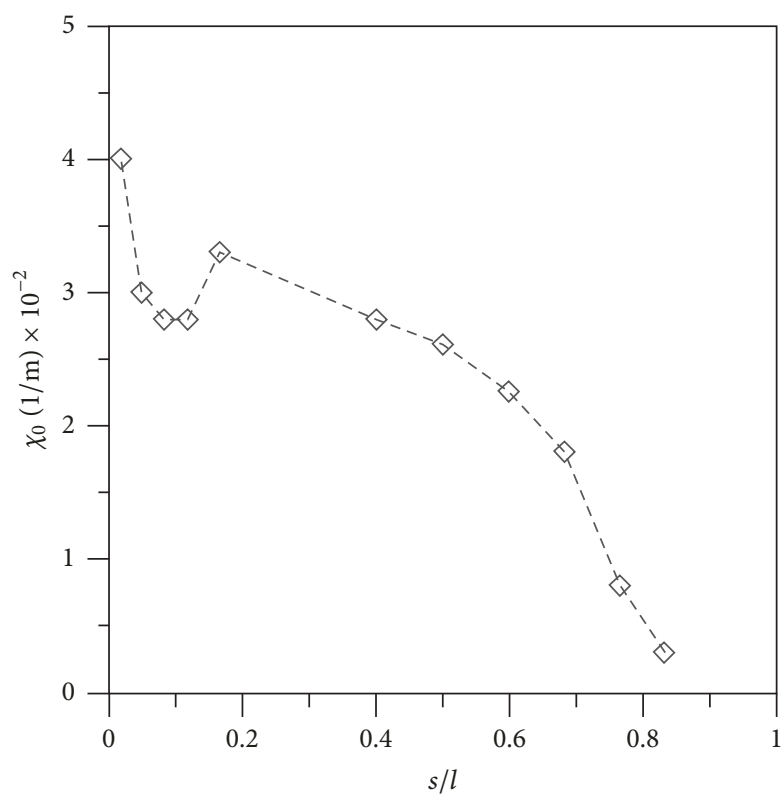

FIGURE 13: Values of the yielding curvature $\chi_{0}$ of the messenger cable identified from the static cyclic test. The values are plotted against the nondimensional arc-length coordinate $s / l$ (see also Figure 6).

and to one at the tip (see also Figure 6). The theoretical values of the maximum and minimum bending stiffness of the cross sections have been also calculated through (11) and plotted in Figures 12(a) and 12(b) for comparison purposes. The reference value of $E=200 \mathrm{GPa}$ has been assumed in the calculations for the Young modulus of the steel wires.

First of all, it can be observed that over a wide central region of the strand the identified stiffness values $E I_{\max }$ and $E I_{\text {min }}$ tend to be constant and equal, respectively, to the values $E I_{\max }=25 \mathrm{Nm}^{2}$ and $E I_{\min }=2.5 \mathrm{Nm}^{2}$. These values, hence, can be considered as representative of the cable behaviour far from the boundaries and compared with the results of (11). The latter, indeed, are derived from a discrete cable model neglecting the effects of the boundary conditions. Equation (11) significantly overestimates the value of $E I_{\max }$, with a difference between theoretical and identified values of about $55 \%$. The discrepancy between the theoretical (11) and identified value of $E I_{\min }$, instead, is in the order of $5 \%$.

The differences between the identified and the theoretical values of the parameter $E I_{\max }$ can be partially due to the effect of the tangential compliance of the internal contact surfaces between the wires, which is neglected in the model at the basis of (11). Indeed, preliminary results presented by one of the authors in [43] show that accounting for the interwire tangential compliance within the classic framework of the Hertzian contact theory can actually reduce the value of the maximum bending stiffness, in both taut and slack singlelayer strands.

From Figures 12(a) and 12(b), the occurrence of a "boundary layer" region near to the clamp can also be clearly appreciated. Within this region, both $E I_{\max }$ and $E I_{\min }$ show a rapid increase from their "far-field" constant values to the values of, respectively, $40 \mathrm{Nm}^{2}$ and $5 \mathrm{Nm}^{2}$ identified at a distance $s=5 \mathrm{~mm}$ from the clamp. The presence of the clamping device can intuitively explain the occurrence of this boundary region, whose length $\left(l_{B 1}\right)$ can be estimated from these figures as $l_{B 1}=0.16 l$ (where $l$ is the length of the strand).

A second boundary layer region, near to the tip of the strand and with length approximately equal to $l_{B 2}=0.23 l$, can be also observed from the same two Figures 12 (a) and 12(b). The stiffness parameter $E I_{\max }$ increases up to the same value of $40 \mathrm{Nm}^{2}$ observed near to the clamp. On the other hand, a counterintuitive decreasing of the value of the minimum bending stiffness $E I_{\min }$ is observed close to the tip. This could also be, however, a spurious effect due to fact that the identification procedure in this region is based on the less accurate moment-curvature diagrams of sections 10 and 11 (see also Figure 11).

Figure 13 shows the yielding curvature parameter, $\chi_{0}$, as a function of nondimensional abscissa $s / l$. Within the central region of the strand, that is, approximately for $0.2 \leq s / l \leq 0.6$, the parameter $\chi_{0}$ linearly varies within the range of values from 0.02 to $0.03(1 / \mathrm{m})$. In the boundary region near the clamp, the values of $\chi_{0}$ exhibit a rapid increase, which can be explained by recalling that the radial pressures exerted by the clamp on the messenger cable tend to contrast the relative sliding between the wires. This restraining effect tends to increase toward the clamp, where the interwire displacement can be assumed to be strictly equal to zero, leading to the limit theoretical condition of $\chi_{0} \rightarrow+\infty$, for $s / l \rightarrow 0$. Finally, a third region can be identified from Figure 13 . For $s / l \geq 0.6$, the curve $\chi_{0}(s / l)$ shows a variation of the slope with respect to the one characterizing the central region of the strand, leading to a rapid decrease of $\chi_{0}$ from about $0.02(1 / \mathrm{m})$ toward the zero.

The distribution of the identified values of $\chi_{0}$ along the length of the strand, hence, leads to two main observations. On the one hand, it confirms the existence of a boundary layer region in the proximity of the clamp, already suggested by the analysis of the identified values of the stiffness parameters $E I_{\max }$ and $E I_{\min }$. On the other one, it can suggest a dependence of the parameter $\chi_{0}$ on the maximum curvature $\chi_{\max }$ reached during the hysteresis loops (see also Figures $7-11)$. To further investigate this effect, the identified values of the parameter $\chi_{0}$ are plotted in Figure 14 against $\chi_{\max }$ for all the sections outside the boundary layer region near the clamp, that is, sections $6-11$, which are characterized by $s / l \geq 0.2$.

The nonlinear relation highlighted in Figure 14 points out that in the cable there has been dissipation mechanism of different importance depending on the vibration amplitude. Given the relatively simple hysteretic model herein adopted, this is reflected in a smaller value of $\chi_{0}$ at the decrease of $\chi_{\max }$ in order to correctly reproduce the energy dissipation.

In the light of the rich and complex scenario coming from the analysis of the cross-sectional behaviour of the messenger cable, some simplifications need to be introduced in order to achieve a good trade-off between the accuracy and complexity of the model.

To this aim, a fundamental distinction between the boundary layer and far-field regions of the messenger cable is introduced by defining two different prototype sections. The latter are meant to be sufficiently representative, respectively, of the behaviour of the strand in the proximity of the 
TABLE 3: Constitutive parameters of the cross-sectional hysteretic model for the prototype sections in the far-field and boundary layer regions of the messenger cable.

\begin{tabular}{lcccc}
\hline & $E I_{\max }\left(\mathrm{Nm}^{2}\right)$ & $E I_{\min }\left(\mathrm{Nm}^{2}\right)$ & $\chi_{0}\left(\mathrm{~m}^{-1}\right) \times 10^{-2}$ & $\sigma$ \\
\hline "Far-field" section & 25 & 2.5 & 3.0 & 1 \\
"Boundary-layer" section & 40 & 5.0 & 15.0 & 1 \\
\hline
\end{tabular}

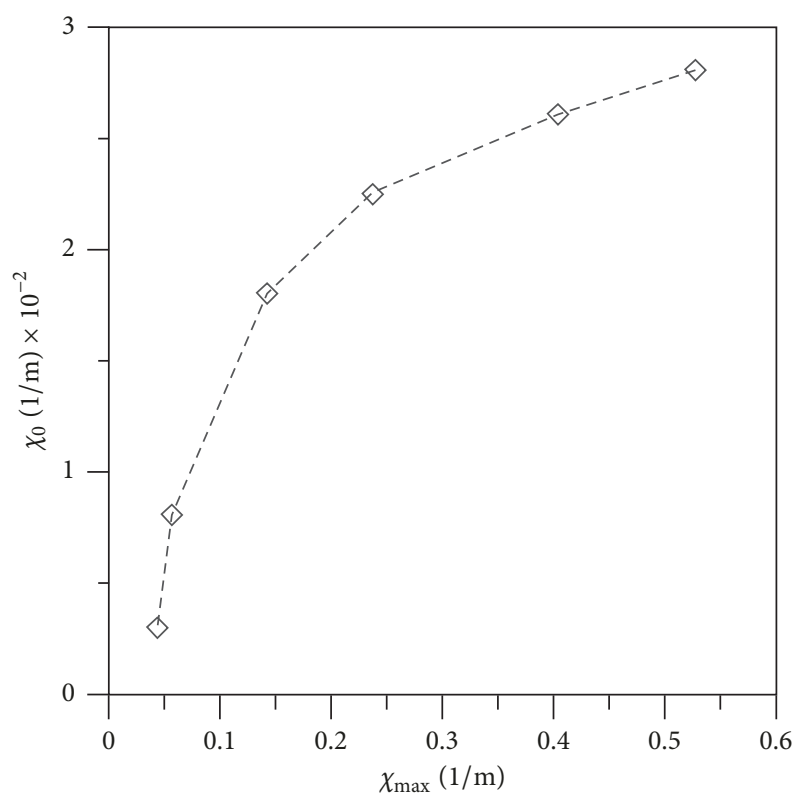

FIGURE 14: Relation between the identified values of the yielding curvature $\chi_{0}$ of the cross sections of the messenger cable and the maximum $\chi_{\max }$ curvature measured during the experimental hysteresis cycles.

restraints or far from them. The constitutive parameters adopted for these two sections are listed in Table 3.

Once this simplification is introduced, the lengths of the two boundary regions in the proximity of the clamp and of the tip of the cable (herein denoted, respectively, as $l_{B 1}$ and $l_{B 2}$ ) should be regarded as two additional parameters of the model. The analysis of the results of the cross-sectional identification procedure, however, gives an indication on the possible extent of the boundary regions, which can be assumed in the range of $10-25 \%$ of the length of the specimen.

\section{Capabilities of the Proposed Modelling Strategy}

The proposed modelling strategy is based on a local description of the cyclic bending behaviour through the Bouc-Wen hysteretic model and on the adoption of a classic structural theory for the messenger cable. The capability of the proposed modelling strategy to capture the response of Stockbridge dampers will be presented in terms of the global behaviour of the damper reported by Sauter and Hagedorn for the experimental cyclic tests already adopted for the modelling of the local behaviour $[18,19]$. First, the comparison will be in terms of the results from quasi-static cyclic tests, then in

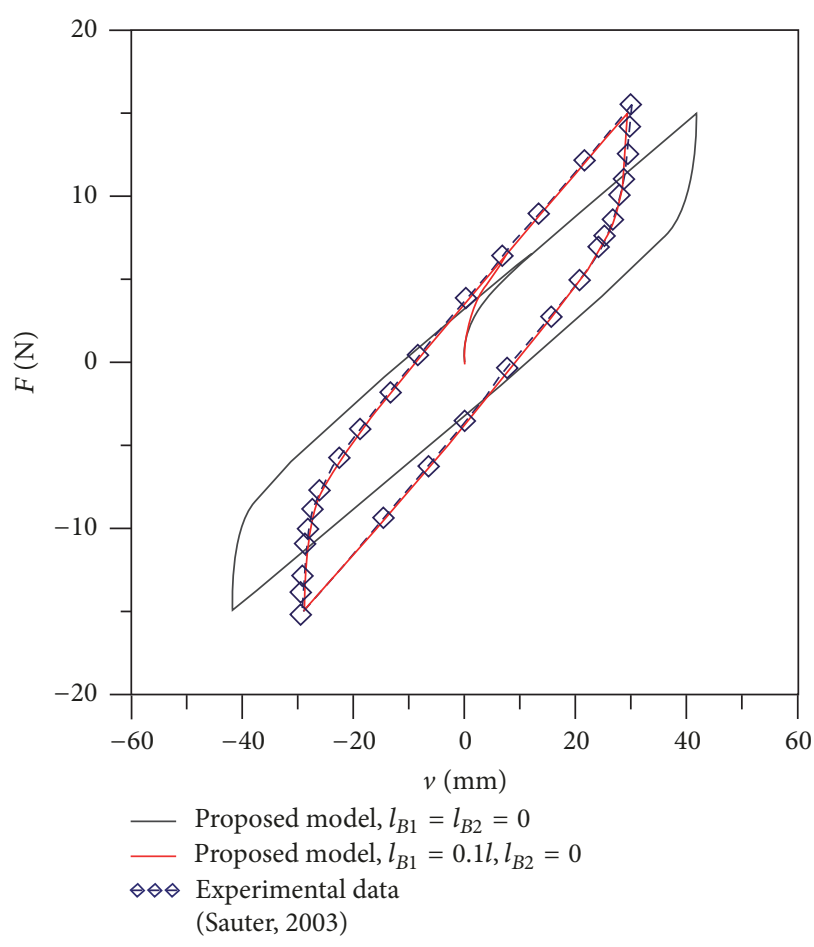

Figure 15: Cyclic static test. Comparison between the results of the proposed cable model and the experimental data reported by Sauter [18]. The results of the proposed model are plotted for two different choices of the boundary layer lengths $l_{B 1}$ and $l_{B 2}$.

terms of the Stockbridge damper response in the frequency domain.

5.1. Quasi-Static Response. The bending response of the specimen tested by Sauter and Hagedorn $[18,19]$ has been numerically simulated by solving (16) for a prescribed vertical load, cyclically varying between the values of $\pm 15 \mathrm{~N}$. The numerical simulations have been carried out by assuming the constitutive parameters listed in Table 3 for the boundary layer and far-field cross sections and a total number of 30 Gauss-Lobatto integration points have been adopted to evaluate the integrals in (16). Different grids of points have been also preliminary considered in order to exclude the dependency of the simulation results on the chosen integration grid.

Figure 15 shows a comparison between the experimentally determined load-displacement curve and the results of the proposed hysteretic model. The numerical results are shown for two different values of the boundary layer length $l_{B 1}: l_{B 1}=0$, and $l_{B 1}=0.1$, with $l$ as in Figure 6 . In both cases, the length of the boundary layer close to the 


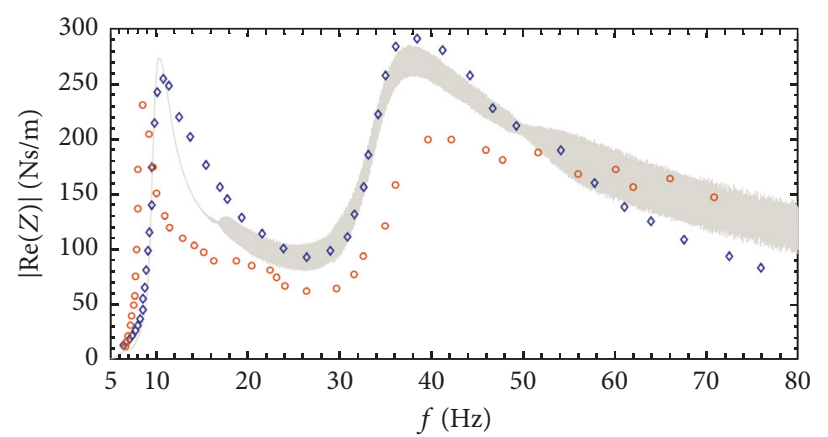

$$
\begin{array}{ll} 
& \text { Proposed model } \\
\diamond \quad \text { Experimental data } \\
\text { - Sauter's model }
\end{array}
$$

FIGURE 16: Real part of the impedance curve of the Stockbridge damper tested on a shaker for sweep tests in frequency with an imposed vertical translation of the clamp having a constant value of the clamp velocity equal to $0.2 \mathrm{~m} / \mathrm{s}$. Comparison between the results of the proposed model, the experimental data and the hysteretic model proposed by Sauter and Hagedorn (Sauter's model). The experimental data and the results of Sauter's model are from [18].

tip of the specimen has been assumed equal to zero (i.e., $\left.l_{B 2}=0\right)$. Preliminary calculations, indeed, have shown that varying the length of the boundary layer close to the tip from zero up to the $20 \%$ of the length of the specimen does not significantly affect the overall response of the strand. This was expected, since, due to the particular loading conditions and the resulting triangular bending moment distribution, the overall response of the cable turns out to be mostly controlled by the hysteretic response of the cross sections in the neighbourhood of the clamp.

The structural response reported in Figure 16, computed from the identified local (sectional) behaviour, is in excellent agreement with the experimental one once the flexural boundary layer at the clamp has been accounted for.

5.2. Dynamic Response. In this section, the general modelling procedure described in the previous sections is used to characterize the dynamic response of a specimen of a symmetric Stockbridge damper that was experimentally tested by Sauter and Hagedorn $[18,19]$ on a shaker for an imposed vertical translation of the clamp: sweep tests in frequency at constant value of the clamp velocity (equal to $0.2 \mathrm{~m} / \mathrm{s}$ ). Being the experimental test of the type at constant velocity, there is not a single value of amplitude of the motion, since this depends on the frequency. Due to the nonlinearity of the Stockbridge response, this last aspect makes achieving a satisfactory matching with the experimental data even more challenging.

The efficiency of Stockbridge dampers in damping aeolian vibrations is one important issue in power line design. One of the codified [41] ways to measure the damper efficiency is by first evaluating the damper mechanical impedance at the clamp through translational tests on an electrodynamic exciter. Then, the steady state amplitudes of the aeolian vibrations, and the related strains on the real span, can be computed resorting to the energy balance principle (EBP) for the energy input from the wind and the energy dissipated by the cable + dampers assemblage. The damper is considered efficient if the computed strains are lower than the allowable ones in all of the aeolian vibrations frequency range. Although not perfect (see, e.g., $[44,45]$ ), the method is well accepted in the practice. Another use of the impedance curve found on a shaker is to prove that production samples have similar dynamic response as dampers qualified in the design tests.

In order to carry out the assessment, the equations of motion for the general formulation presented in Section 2.2 are modified for the particular case at hand of symmetric Stockbridge damper, as it follows. For a symmetric Stockbridge damper subjected only to a vertical translation of the clamp, the dynamic equilibrium equation for the clamp becomes

$$
\begin{aligned}
F_{c} & =\left(2 m_{d}+m_{c}\right) \ddot{w}_{c}+2 m_{d} \ddot{v}_{d}-2 m_{d} e_{G d} \ddot{\varphi}_{d} \\
M_{c} & =0
\end{aligned}
$$

along with the following equations of motion for the attached inertial bodies:

$$
\begin{aligned}
m_{d} \ddot{v}_{d}-m_{d} e_{G d} \ddot{\varphi}_{d}+F_{d} & =-m_{d} \ddot{w}_{c} \\
-m_{d} e_{G d} \ddot{v}_{d}+\left(e_{G d}^{2} m_{d} \ddot{\varphi}_{d}+I_{G d}\right)+M_{d} & =m_{d} e_{G d} \ddot{w}_{c} .
\end{aligned}
$$

Equations (18) have been numerically integrated for a sinusoidal motion, in vertical direction, of the clamp and the reaction of the clamp has been subsequently evaluated through the first of the two equations (17). During the test, sinusoid frequency undergoes a linear sweep from 0 to $100 \mathrm{~Hz}$ during a total duration of $200 \mathrm{~s}$. A time step $\Delta t=0.0001 \mathrm{~s}$ was adopted in the numerical analyses in association with a Newmark constant acceleration time-stepping strategy. The other parameters of the Stockbridge damper model are listed in Table 4.

Figure 16 presents the comparison of the results obtained from the proposed modelling strategy (which adopts only two different identified hysteretic laws), the one by Sauter [18] which employs more than fifteen identified Masing laws, 


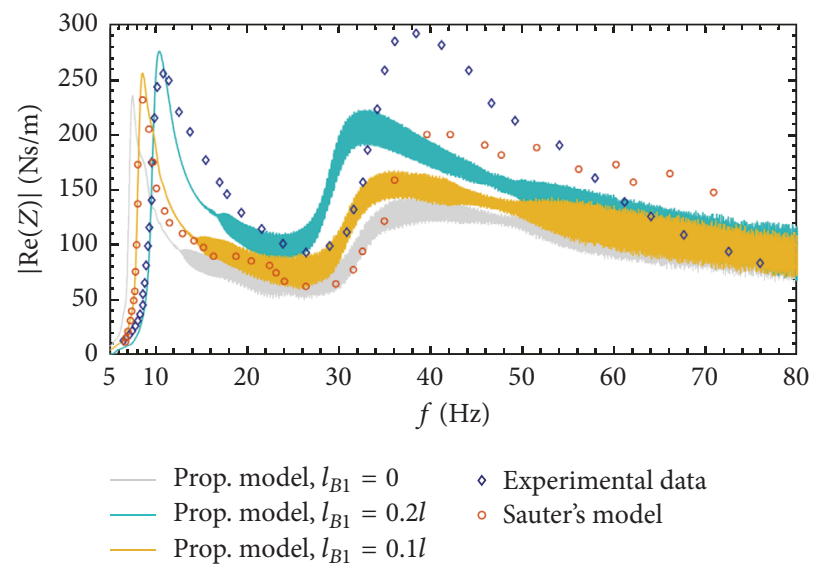

FIGURE 17: Real part of the impedance curve of the Stockbridge damper tested on a shaker for sweep tests in frequency with an imposed vertical translation of the clamp having a constant value of the clamp velocity equal to $0.2 \mathrm{~m} / \mathrm{s}$. Comparison between the results of the proposed model, the experimental data, and the hysteretic model proposed by Sauter and Hagedorn (Sauter's model). The experimental data and the results of Sauter's model are from [18]. The results of the proposed model are plotted for three different choices of the boundary layer lengths $l_{B 1}$ and $l_{B 2}$.

TABLE 4: Geometric and inertial properties of the Stockbridge damper. The data are from [18].

\begin{tabular}{lccc}
\hline$l(\mathrm{~m})$ & $e_{\mathrm{Gd}}(\mathrm{m})$ & $m_{d}(\mathrm{~kg})$ & $I_{\mathrm{Gd}}\left(\mathrm{kgm}^{2}\right)$ \\
\hline 0.1875 & 0.0325 & 0.856 & 0.001814 \\
\hline
\end{tabular}

and the experimental test; in terms of the real part of the impedance function, $Z$, of the damper, this is defined as the ratio between the force at the clamp over the clamp velocity. The real parts of the impedance can be used to characterize the dissipated power. The results shown have been obtained after calibration of the boundary layer lengths $l_{B 1}$ and $l_{B 2}$. The values identified for these model parameters are quite close to the one already presented in relation to the outcome of the quasi-static cyclic tests (and of the experimentally identified boundary layers in Figure 12): $l_{B 1}=0.2 l ; l_{B 2}=0.08 l$.

On the basis of Figure 16, we can notice the good performance of the proposed modelling strategy that is able to capture the position and the maximum value, for both resonances (the one at about $11 \mathrm{~Hz}$ and the one at about $38 \mathrm{~Hz}$ ). The match over the full frequency range is very good also in light that the curves were plotted with a linear scale (not a dB scale, as it is customary for frequency response functions) on the vertical axis. The numerical model proposed by Sauter appears less accurate in reproducing the experimental data, shifting the position of the first resonance peak to lower frequencies by about 30\%, the position of the second resonance to higher frequencies by about $10 \%$, and significantly underpredicting the maximum value of the impedance function (more notably for the second resonance).

As mentioned, the values of the model parameters $l_{B 1}, l_{B 2}$ were calibrated. In order to highlight the importance of the first parameter, Figure 17 depicts how the impedance function is affected by the value of $l_{B 1}\left(l_{B 2}\right.$ is always assumed equal to zero for these results). This parameter affects the resonance related to both modes due to couplings between the modes. The increase of this parameters leads to an increase of both resonance peaks and to a reduction of their separation with a shift of both toward the central frequency.

Comparison of the curve for $l_{B 1}=0.2 l$ and $l_{B 2}=0$ in Figure 17 with the one for $l_{B 1}=0.2 l$ and $l_{B 2}=0.08 l$ in Figure 16 shows that the second parameter mainly affects the resonance related to second mode, shifting the resonance peak to higher frequencies and higher values as the length of this boundary layer zone increases.

\section{Conclusions}

In this work, a simple mechanical model of a Stockbridge damper has been presented. The model is based on a beamlike description of the messenger cable and on the crosssectional inelastic nonlinear cyclic bending behaviour.

Starting from a description of the interwire sliding process, the classic Bouc-Wen hysteretic model has been recognized as adequate to represent the cyclic bending behaviour of the cross sections.

The model parameters are identified from experimental results available in literature and used at the local (crosssectional level) to characterize the mechanical behaviour of the messenger cable. The descending global behaviour of the Stockbridge damper compares very favourably with the experimental results in terms of the impedance function at the clamp and allows for the confident used of the proposed model inside the assessing process of full lines.

The important role of the end zones of the messenger cable, where a boundary layer like transition has been found for the bending stiffness of the messenger cable cross section, has been highlighted, along with its effects on the global response of the damper. Without the definition of the length of these end zones the match with the experimental results is only partially satisfactory. These lengths have been considered as additional model parameters. 
As a final thought, the model depends on some parameters that can vary over the life of the Stockbridge damper (e.g., the aging of the messenger cable can affect wear and change the interwire sliding process). As for all the other Stockbridge models to the knowledge of the authors, this aspect has not been pursued in this work.

\section{Conflicts of Interest}

The authors declare that they have no conflicts of interest.

\section{Acknowledgments}

This work has been partially supported by MIUR (Italian Ministry of Education, University and Research) under the project "PRIN 2015-2018-Identification and Monitoring of Complex Structural Systems”.

\section{References}

[1] P. Hagedorn, "On the computation of damped wind-excited vibrations of overhead transmission lines," Journal of Sound and Vibration, vol. 83, no. 2, pp. 253-271, 1982.

[2] M. Markiewicz, "Optimum dynamic characteristics of stockbridge dampers for dead-end spans," Journal of Sound and Vibration, vol. 188, no. 2, pp. 243-256, 1995.

[3] G. Diana et al., "Modelling of aeolian vibrations of a single conductor plus damper: assessment of technology," Electra, vol. 223, pp. 28-36, 2005.

[4] H. Wolf, B. Adum, D. Semenski, and D. Pustaić, "Using the Energy Balance Method in estimation of overhead transmission line Aeolian vibrations," Strojarstvo, vol. 50, no. 5, pp. 269-276, 2008.

[5] O. Barry, R. Long, and D. C. D. Oguamanam, "Rational Damping Arrangement Design for Transmission Lines Vibrations: Analytical and Experimental Analysis," Journal of Dynamic Systems, Measurement, and Control, vol. 139, no. 5, Article ID 051012, 2017.

[6] T. Argentini, L. Rosa, and A. Zasso, "Wind-induced vibrations of the stay cables of a roundabout flyover: Assessment, repair, and countermeasures," Journal of Performance of Constructed Facilities, vol. 30, no. 6, Article ID 04016046, 2016.

[7] C. Klinger, "Failures of cranes due to wind induced vibrations," Engineering Failure Analysis, vol. 43, pp. 198-220, 2014.

[8] G. H. Stockbridge, "Overcoming vibrations in transmission cables," Electric World, vol. 86, pp. 1304-1305, 1925.

[9] EPRI Electric Research Power Institute, Transmission Line Reference Book: Wind-Induced Conductor Motion, 2006.

[10] R. Claren and G. Diana, "Mathematical Analysis of Transmission Line Vibration," IEEE Transactions on Power Apparatus and Systems, vol. 88, no. 12, pp. 1741-1771, 1969.

[11] H. Wagner, V. Ramamurti, R. V. R. Sastry, and K. Hartmann, "Dynamics of stockbridge dampers," Journal of Sound and Vibration, vol. 30, no. 2, pp. 207-220, 1973.

[12] O. Barry, D. C. D. Oguamanam, and D. C. Lin, "Aeolian vibration of a single conductor with a Stockbridge damper," Proceedings of the Institution of Mechanical Engineers, Part C: Journal of Mechanical Engineering Science, vol. 227, no. 5, pp. 935-945, 2013.
[13] D. Hartog, Mechanical Vibrations, Dover Publications, New York, NY, USA, 14th edition, 1985.

[14] S. Langlois and F. Legeron, "Prediction of aeolian vibration on transmission-line conductors using a nonlinear time history model - Part I: Damper model," IEEE Transactions on Power Delivery, vol. 29, no. 3, pp. 1168-1175, 2014.

[15] N. Carpineto, W. Lacarbonara, and F. Vestroni, "Hysteretic tuned mass dampers for structural vibration mitigation," Journal of Sound and Vibration, vol. 333, no. 5, pp. 1302-1318, 2014.

[16] I. Pivovarov and O. G. Vinogradov, "One application of Bouc's model for non-linear hysteresis," Journal of Sound and Vibration, vol. 118, no. 2, pp. 209-216, 1987.

[17] R. Bouc, "Modèle mathématique d'hystérésis," Acustica, vol. 21, pp. 16-25, 1971.

[18] D. Sauter, Modeling the dynamic characteristics of slack wire cables in Stockbridge dampers, Technische Universitat Darmstadt (Dissertation), Darmstadt, Germany, 2003.

[19] D. Sauter and P. Hagedorn, "On the hysteresis of wire cables in Stockbridge dampers," International Journal of Non-Linear Mechanics, vol. 37, no. 8, pp. 1453-1459, 2002.

[20] N. Barbieri and R. Barbieri, "Dynamic analysis of stockbridge damper," Advances in Acoustics and Vibration, vol. 2012, Article ID 659398, 8 pages, 2012.

[21] N. Barbieri, R. Barbieri, R. A. da Silva, M. J. Mannala, and L. D. S. V. Barbieri, "Nonlinear dynamic analysis of wire-rope isolator and Stockbridge damper," Nonlinear Dynamics, vol. 86, no. 1, pp. 501-512, 2016.

[22] X. Luo, L. Wang, and Y. Zhang, "Nonlinear numerical model with contact for Stockbridge vibration damper and experimental validation," Journal of Vibration and Control, vol. 22, no. 5, pp. 1217-1227, 2014.

[23] H. Goldstein, Classical Mechanics, Addison-Wesley Publishing Company, 1959.

[24] A. Bedford, Hamilton's Principle in Continuum Mechanics, Pitman Publishing, London, UK, 1985.

[25] F. Foti and L. Martinelli, "Mechanical modeling of metallic strands subjected to tension, torsion and bending," International Journal of Solids and Structures, vol. 91, pp. 1-17, 2016.

[26] C. B. Rawlins, Analytical Elements of Overhead Conductor Fabrication, Fultus Books, California, Calif, USA, 2005.

[27] M. Frigerio, P. B. Buehlmann, J. Buchheim et al., "Analysis of the tensile response of a stranded conductor using a 3D finite element model," International Journal of Mechanical Sciences, vol. 106, pp. 176-183, 2016.

[28] F. Foti and L. Martinelli, "An analytical approach to model the hysteretic bending behavior of spiral strands," Applied Mathematical Modelling: Simulation and Computation for Engineering and Environmental Systems, vol. 40, no. 13-14, pp. 6451-6467, 2016.

[29] A. Cardou, "Taut helical strength bending stiffness," 2006, http://www.utfscience.deI/.

[30] K. O. Papailiou, "On the bending stiffness of transmission line conductors," IEEE Transactions on Power Delivery, vol. 12, no. 4, pp. 1576-1583, 1997.

[31] F. Foti, A corotational beam element and a refined mechanical model for the nonlinear dynamic analysis of cables [Ph.D dissertation], Politecnico di Milano, Milan, Italy, 2013.

[32] M. Ismail, F. Ikhouane, and J. Rodellar, "The hysteresis BoucWen model, a survey," Archives of Computational Methods in Engineering: State-of-the-Art Reviews, vol. 16, no. 2, pp. 161-188, 2009. 
[33] M. Domaneschi, "Simulation of controlled hysteresis by the semi-active Bouc-Wen model," Computers \& Structures, vol. 106-107, pp. 245-257, 2012.

[34] F. Ikhouane, J. Rodellar, and J. E. Hurtado, "Analytical characterization of hysteresis loops described by the Bouc-Wen model," Mechanics of Advanced Materials and Structures, vol. 13, no. 6, pp. 463-472, 2006.

[35] F. Ikhouane, V. Manosa, and J. Rodellar, "Dynamic properties of the hysteretic Bouc-Wen model," Systems and Control Letters, vol. 56, no. 3, pp. 197-205, 2007.

[36] F. Ikhouane, J. E. Hurtado, and J. Rodellar, "Variation of the hysteresis loop with the Bouc-Wen model parameters," Nonlinear Dynamics, vol. 48, no. 4, pp. 361-380, 2007.

[37] M. A. Crisfield, "Nonlinear finite element analysis of solids and structures," in Advanced Topics, vol. 1, Wiley and Sons, New York, NY, USA, 1997.

[38] M. Petrangeli and V. Ciampi, "Equilibrium based iterative solutions for the non-linear beam problem," International Journal for Numerical Methods in Engineering, vol. 40, no. 3, pp. 423437, 1997.

[39] P. K. V. V. Nukala and D. W. White, "Variationally consistent state determination algorithms for nonlinear mixed beam finite elements," Computer Methods Applied Mechanics and Engineering, vol. 193, no. 33-35, pp. 3647-3666, 2004.

[40] M. Quagliaroli, From bidimensional towards monodimensional modeling of sound and damaged reinforced concrete structures [Ph.D. dissertation], Politecnico di Milano, Milan, Italy, 2014.

[41] IEEE Power Engineering Society, "Guide on the measurement of the performance of aeolian vibration dampers for single conductors," IEEE Std, pp. 664-1993, 1993.

[42] F. Foti and L. Martinelli, "A unified analytical model for the selfdamping of stranded cables under aeolian vibrations," Journal of Wind Engineering and Industrial Aerodynamics, vol. 176, pp. 225-238, 2017.

[43] F. Foti, "A corotational finite element to model bending vibrations of metallic strands," in Proceedings of the ECCOMAS Congress 2016, V. Papadopoulos, G. Stefanou, and V. Plevris, Eds., pp. 4455-4466, June 2016.

[44] G. Diana, A. Cigada, M. Belloli, and M. Vanali, "Stockbridgetype damper effectiveness evaluation: Part I - Comparison between tests on span and on the shaker," IEEE Transactions on Power Delivery, vol. 18, no. 4, pp. 1462-1469, 2003.

[45] G. Diana, A. Manenti, C. Pirotta, and A. Zuin, "Stockbridgetype damper effectiveness evaluation: part II - The influence of the impedance matrix terms on the energy dissipated," IEEE Transactions on Power Delivery, vol. 18, no. 4, pp. 1470-1477, 2003. 


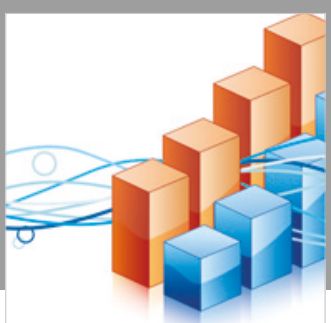

Advances in

Operations Research

\section{-n-m}
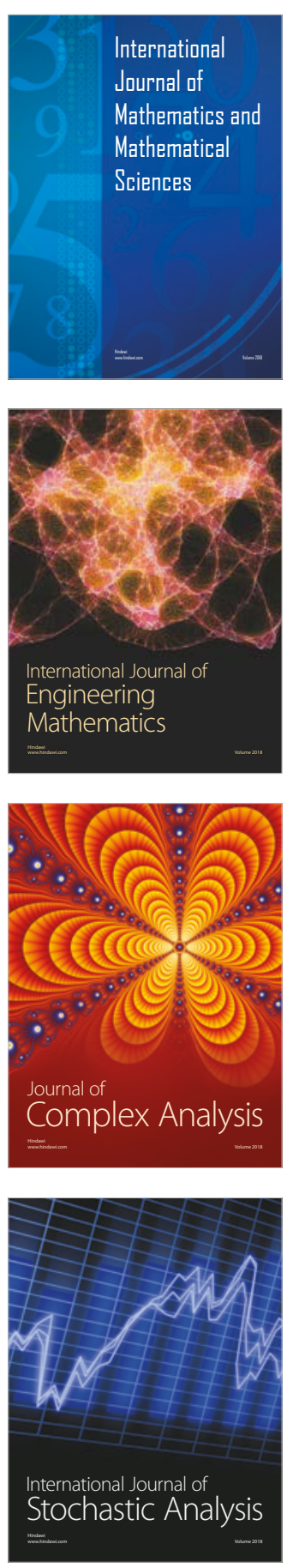
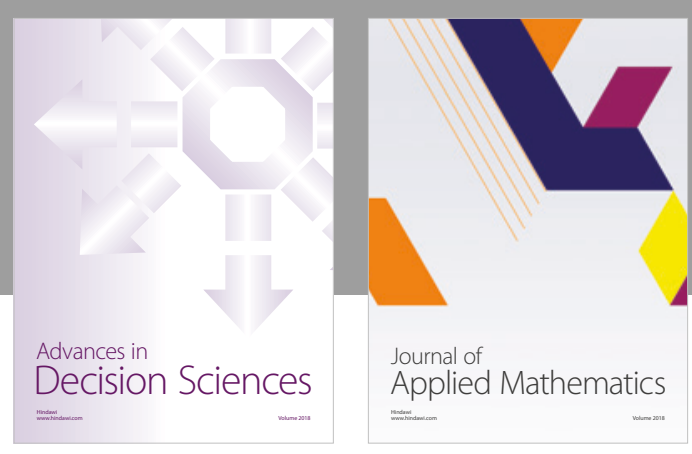

Journal of

Applied Mathematics
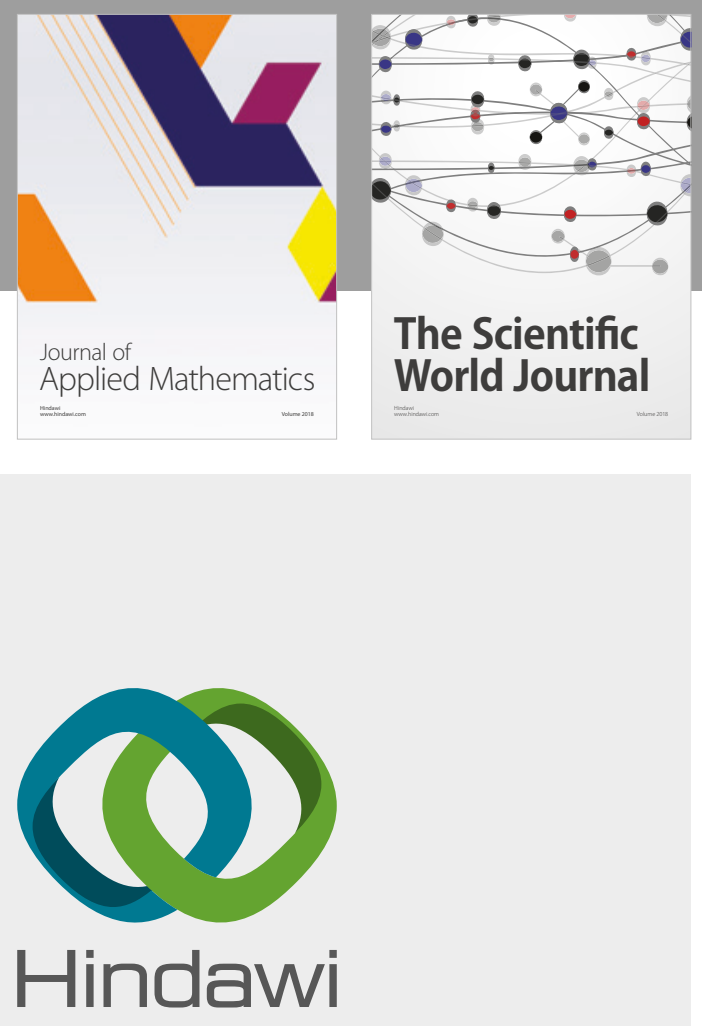

Submit your manuscripts at

www.hindawi.com

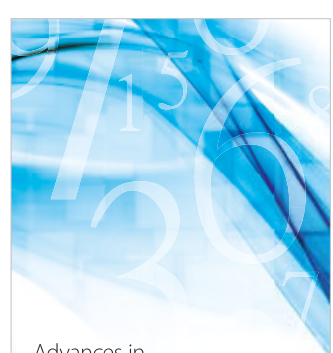

Advances in
Numerical Analysis
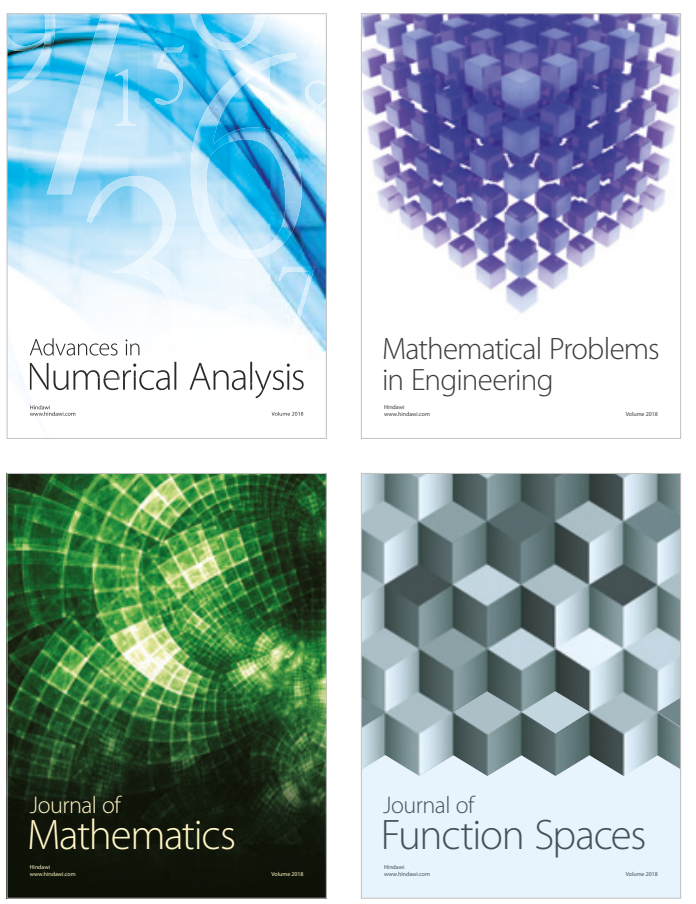

Mathematical Problems in Engineering

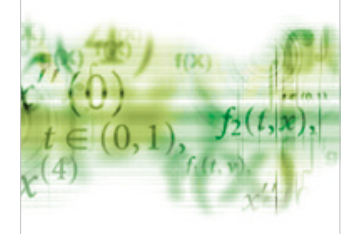

International Journal of

Differential Equations

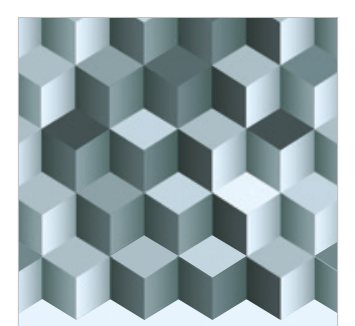

Journal of

Function Spaces

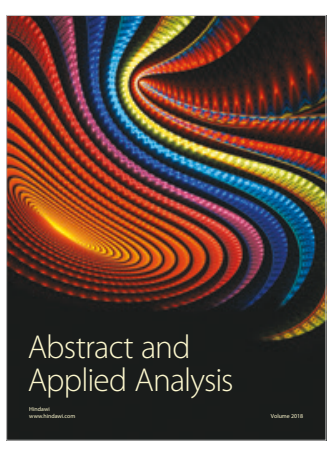

The Scientific

World Journal

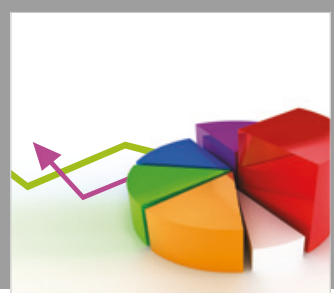

Journal of

Probability and Statistics
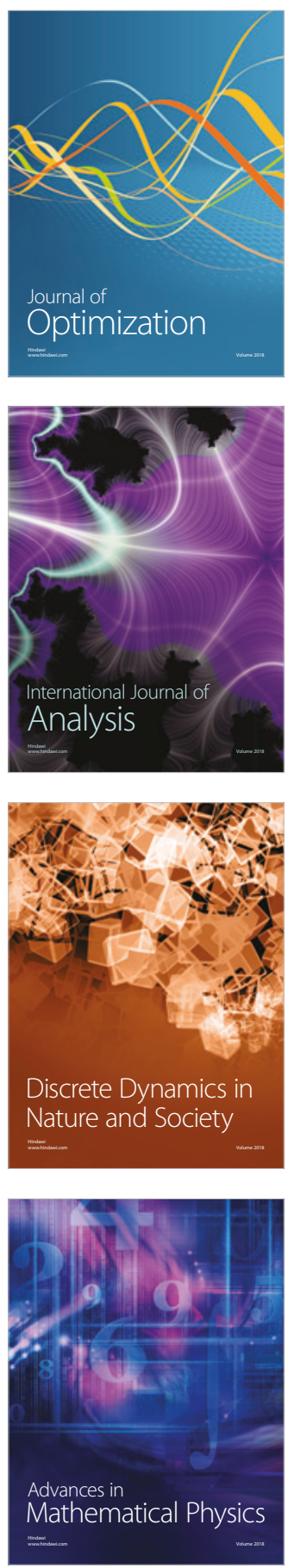